UNIVERSIDADE ESTADUAL PAULISTA “JÚLIO DE MESQUITA FILHO” FACULDADE DE MEDICINA

GUSTAVO HIDEKI KAWANAMI

\begin{abstract}
PREDITORES DE INCORREÇÃO NA PRESCRIÇÃO DE ANTIMICROBIANOS NO HOSPITAL ESTADUAL BAURU (BAURU, SÃO PAULO).
\end{abstract}

Dissertação apresentada ao Programa de PósGraduação em Doenças Tropicais da Faculdade de Medicina de Botucatu, Universidade Estadual Paulista, para obtenção do título de Mestre.

Orientador: Prof.Dr. Carlos Magno C. B. Fortaleza

BOTUCATU - SP

2010 
UNIVERSIDADE ESTADUAL PAULISTA “JÚLIO DE MESQUITA FILHO” FACULDADE DE MEDICINA

\author{
GUSTAVO HIDEKI KAWANAMI
}

\title{
PREDITORES DE INCORREÇÃO NA PRESCRIÇÃO DE ANTIMICROBIANOS NO HOSPITAL ESTADUAL BAURU (BAURU, SÃO PAULO).
}

Dissertação apresentada ao Programa de PósGraduação em Doenças Tropicais da Faculdade de Medicina de Botucatu, Universidade Estadual Paulista, para obtenção do título de Mestre.

Orientador: Prof.Dr. Carlos Magno C. B. Fortaleza

BOTUCATU - SP

2010 


\section{DEDICATÓRIA}

Para meus pais, irmã e esposa

"What greater thing is there for human souls than to feel that they are joined for life to be with each other in silent unspeakable memories."

(George Eliot) 


\section{AGRADECIMENTOS}

A Valéria Drumond Nagem Aragão, por me apresentar, ensinar e fazer gostar da vida profissional que vivo hoje.

A Edson Carvalho de Melo, pelo encorajamento, por assumir minhas responsabilidades nos momentos em que foi preciso me ausentar.

Aos colegas médicos residentes em Infectologia da Faculdade de Medicina de Botucatu entre os anos de 2004 e 2006, Ana Flávia Bonini, Gabriela Gryninger, Ligia Castellon Figueiredo, Paulo Afonso Martins Abati, Patricia Bernardinelli Martino, Taylor Endrigo Toscano Olivo e Ricardo de Souza Cavalcante, pela troca de experiências, pelo apoio e pela amizade.

Ao professor e amigo Carlos Magno, que mesmo quando eu já havia desistido, sempre conseguia me ensinar algo.

A Hideo Kawanami, meu pai, aquele que entre tudo, sempre me indicou o caminho.

A Olinda Keiko Shoyama Kawanami, minha mãe, que a cada dia me ensinou a trilhar o caminho.

A Adriana, minha esposa, pela sua inata capacidade de me acalmar e me trazer de volta ao mundo real. 


\section{EPÍGRAFE}

"The future of humanity and microbes will likely evolve as episodes of our wits versus their genes"

Jonathan Laderberg

(Science 2000; 288: 281-93) 
SUMÁRIO

Seção

Página

Resumo

7

Abstract

9

Introdução geral

Referências da introdução 32

Objetivos 43

Artigo 44

Conclusão 71

Apêndice 72 


\section{RESUMO}

O uso incorreto de antimicrobianos nos hospitais tem impacto negativo sobre o prognóstico dos pacientes e está associado à emergência e disseminação de microorganismos multidroga-resistentes. Programas de supervisão do uso de antimicrobianos (PSA) têm sido instituídos com o objetivo de promover a qualidade da prescrição desses medicamentos no hospital. Nesse contexto, a identificação de padrões de incorreção na prescrição de antimicrobianos é uma ferramenta valiosa para os PSA, pois permite o direcionamento de ações educativas e restritivas. Com esse objetivo, foi realizado um estudo no Hospital Estadual Bauru, um hospital de ensino de 285 leitos ligado à Faculdade de Medicina de Botucatu, Universidade Estadual Paulista. O hospital possui um PSA ativo desde sua inauguração, em 2002. Foram selecionadas $25 \%$ das solicitações de antimicrobianos de uso parenteral do ano de 2005. Excluíram-se as solicitações para uso profilático. Todas as demais foram submetidas a análise, utilizando uma versão modificada de critérios clássicos propostos por Kunin \& Jones. Análises uni e multivariadas foram realizadas para identificar preditores para incorreção em geral e para erros específicos. Prescrições consideradas "apropriadas" ou "provavelmente apropriadas" foram selecionadas como controles em todas as etapas do estudo. Em 963 solicitações incluídas no estudo, 34,6\% apresentavam alguma incorreção. Os preditores gerais de erro foram: prescrição em fins de semana ou feriados $(O R=1,67, \quad I C 95 \%=1,20-2,28, \quad p=0,002)$, pacientes de Unidades de 
Terapia Intensiva(OR=1,57, IC95\%=1,11-2,23, $p=0,01)$, infecção peritoneal $(\mathrm{OR}=2,15, \quad \mathrm{IC} 95 \%=1,27-3.65, \quad \mathrm{p}=0,004)$ ou de trato urinário $(\mathrm{OR}=1,89$, IC95\%=1,25-2,87, $p=0,002)$, terapia com combinação de dois ou mais antimicrobanos $(\mathrm{OR}=1,72, \mathrm{IC} 95 \%=1,15-2,57, \mathrm{p}=0,008)$ e prescrições incluindo penicilinas $(\mathrm{OR}=2,12, \mathrm{IC} 95 \%=1,39-3,25, \mathrm{p}=0,001)$ ou cefalosporinas de primeira geração $(O R=1,74, I C 95 \%=1,01-3,00, p=0,048)$. A consulta prévia a um médico infectologista teve efeito protetor contra prescrição incorreta (OR=0,34, IC95\%=0,24-0,50, $p<0,001)$. Fatores associados a erros específicos de prescrição apresentaram ampla variação entre as diversas análises. No entanto, a consulta com infectologista foi protetora tanto contra uso desnecessário de antimicrobianos use $(\mathrm{OR}=0,04, \mathrm{IC} 5 \%=0,01-0,26, \mathrm{p}=0,001)$ como contra a solicitação de antimicrobianos com espectro insuficiente $(\mathrm{OR}=0,14$, IC95\%=0,030,30, $p=0,01)$. Concluímos que a análise de preditores de incorreção de antimicrobianos nos permitiu a identificação de itens que requeriam pronta intervenção. Também foi possível avaliar positivamente a atuação do PSA, com a demonstração da importância da consulta prévia a um infectologista para assegurar a qualidade das prescrições de antimicrobianos.

Palavras-chave: antimicrobianos, uso racional, prescrição médica, infecção hospitalar, qualidade de assistência à saúde. 


\section{ABSTRACT}

The inappropriate use of antimicrobials in hospitals has negative impact on patients' outcomes and is associated with the emergence and spread of multidrug-resistant microorganisms. Antimicrobial stewardship programs (ASPs) have been instituted in order to improve the quality of prescriptions in hospitals. In this setting, the identification of patterns of inappropriate antimicrobial prescription is a valuable tool that allows ASPs to identify priorities for directing educative/restrictive polices. With this purpose, a study was conducted in Hospital Estadual Bauru (HEB), a teaching hospital with 285 beds affiliated to Faculdade de Medicina de Botucatu (Botucatu School of Medicine), Universidade Estadual Paulista (São Paulo State University). HEB maintains an active ASP since it was opened, in 2002. We selected $25 \%$ of requests of parenteral antimicrobials (RPAs) from year 2005 for analysis. Prescriptions for prophylactic purposes were excluded. All other RPAs were classified according to a modification of classic Kunin \& Jones categories. Univariate and multivariable analysis was performed to identify predictors of general inappropriateness and of specific prescription errors. Prescriptions classified as "aproppriate" or "probably appropriate" were selected as controls in all stages of the study. Among 963 RPAs included in out study, $34.6 \%$ were inappropriate. General predictors of inappropriateness were: prescription on weekends/holidays (OR=1.67, $95 \% \mathrm{Cl}=1.20-2.28, \mathrm{p}=0.002)$, patient from Intensive Care Unit (OR=1.57, $95 \% \mathrm{Cl}=1.11-2.23, \mathrm{p}=0.01)$, peritoneal $(\mathrm{OR}=2.15,95 \% \mathrm{Cl}=1.27-3.65, \mathrm{p}=0.004)$ or urinary tract infection $(\mathrm{OR}=1.89,95 \% \mathrm{Cl}=1.25-2.87, \mathrm{p}=0.002)$, combination 
therapy with two or more antimicrobials $(\mathrm{OR}=1.72,95 \% \mathrm{Cl}=1.15-2.57, \mathrm{p}=0.008)$ and prescriptions including penicillins $(\mathrm{OR}=2.12,95 \% \mathrm{Cl}=1.39-3.25, \mathrm{p}=0.001)$ or first-generation cephalosporins $(\mathrm{OR}=1.74,95 \% \mathrm{Cl}=1.01-3.00, \mathrm{p}=0.048)$. Previous consultation with an infectious diseases (ID) specialist had a protective effect against inappropriate prescription $(\mathrm{OR}=0.34,95 \%=0.24-0.50, \mathrm{p}<0.001)$. Factors independently associated with specific prescription errors varied. However, consultation with ID specialist was protective against both unnecessary antimicrobial use $(\mathrm{OR}=0.04,95 \% \mathrm{Cl}=0.01-0.26, \mathrm{p}=0.001)$ and requests of agents with insufficient spectrum $(\mathrm{OR}=0.14,95 \% \mathrm{Cl}=0.03-0.30, \mathrm{p}=0.01)$. In conclusion, the analysis of factors predictive of inappropriateness in antimicrobial prescription allowed us to identify issues requiring intervention. Also, it provided a positive feedback of the ASP efficacy, demonstrating the importance of previous consultation with an ID specialist to assure the quality of antimicrobial prescriptions.

Key-words: antimicrobials, antimicrobial stewardship, medical prescriptions, healthcare-acquired infections, quality of health care. 


\section{INTRODUÇÃO GERAL}

\section{Breve história do uso irracional de antimicrobianos.}

A descoberta dos antimicrobianos foi a última e mais bem sucedida etapa da chamada "revolução pasteuriana". É um fato conhecido que diversas substâncias com ação anti-infecciosa (mirra, vinho, sais inorgânicos, soja mofada) faziam parte do arsenal terapêutico das febres na China antiga, e estavam incluídas no corpus hipocraticum. ${ }^{2,3}$ No entanto, somente com o advento da teoria microbiana tornou-se possível conhecer nossos pequenos inimigos, e desenvolver agentes que pudessem elimina-los causando danos mínimos ao organismo humano. De fato, somente entre 1880 e 1900, cerca de vinte agentes infecciosos foram descritos e relacionados à etiologia de doenças específicas. $^{4}$

A era antimicrobiana tem início, de fato, com a descoberta da penicilina por Flemming $^{5}$ e das sulfonamidas por Domagk ${ }^{6}$. Os anos que se seguiram testemunharam contínuas descobertas de novos antibióticos, e uma alteração miraculosa no prognóstico de pacientes com infecções graves. ${ }^{7}$ Não é de se espantar, portanto, que logo se desenvolvesse a pressão científica, comercial e mesmo popular para uma prescrição cada vez mais freqüente dos antimicrobianos. $^{8}$

As expectativas sofreram o primeiro golpe no início dos anos 1950, com a emergência e disseminação dos Staphylococcus aureus resistentes à penicilina 
em ambientes hospitalares. Ainda assim, a resistência a antimicrobianos era descrita como um fenômeno quase restrito a laboratórios. Vale citar um artigo de revisão de Eagle \& Saz $(1955)^{8}$ :

"O medo de que, como resultado do uso disseminado de antimicrobianos muitas espécies bacterianas desenvolverão resistência em um grau suficiente para interferir com a eficácia terapêutica, provou-se, até esta data, infundado." (grifo nosso).

Os autores fazem uma ressalva, porém ao $S$. aureus, informando ser essa bactéria a única a desenvolver resistência clinicamente significante, possivelmente associada ao uso de antimicrobianos. Um ano depois, um artigo publicado por Jawetz adota um tom veemente na admoestação contra o uso excessivo de antimicrobianos. ${ }^{7} \mathrm{O}$ autor manifesta espanto com o fato de, após apenas uma década de uso clínico, os antibióticos já representaram uma das drogas mais prescritas nos Estados Unidos. Postula que cerca de $95 \%$ do uso seja incorreto ou desnecessário. Enfatiza a ocorrência de toxicidade, reações alérgicas e a preocupante emergência da resistência bacteriana. Critica o marketing das indústrias farmacêuticas (“..a nova droga... a chegar no mercado americano e nas páginas de propaganda não é realmente nova"), o excessivo uso profilático (“...a maioria das profilaxias é inútil e mal recomendada”), as terapias combinadas ("em muitos casos... não há evidência convincente de que são desejáveis").

As previsões de Jawetz foram comprovadas nos anos seguintes. Quase duas décadas depois, Kunin et $\mathrm{al}^{9}$ avaliaram os padrões de prescrição de 
antimicrobianos no Hospital da Universidade da Virgínia. Identificaram que 52\% das prescrições eram desnecessárias ou incorretas. Os autores recomendaram o desenvolvimento de estratégias para controle do uso de antimicrobianos em hospitais.

Embora os estudos clássicos citados acima tenham sido publicados nas décadas de 1950 e 1970, eles não surtiram qualquer efeito em frear o uso indiscriminado de antimicrobianos. O desenvolvimento da tecnologia médica, com o conseqüente aumento da complexidade dos procedimentos, e o advento dos microorganismos multi-droga-resistentes (MDR) nos hospitais apenas contribuiu para elevar o consumo dos antimicrobianos. Estes passaram a ter vida útil limitada pela rápida emergência de resistência. A indústria farmacêutica conseguiu-se manter um passo adiante, lançando antimicrobianos capazes de superar os mecanismos de resistência. Mas o desenvolvimento de novos antimicrobianos se faz em velocidade cada vez menor. Essa exaustão de nossa capacidade tecnológica pode gerar uma temida "era pós-antibiótica", em que não haverá terapia eficaz contra grande parte dos microorganismos patogênicos. $^{10}$

Nesse contexto, faz-se necessária uma melhor avaliação dos riscos envolvidos na prescrição de antimicrobianos, especialmente no ambiente hospitalar. 


\section{Riscos associados ao uso de antimicrobianos}

Já em 1955, Eagle \& Saz identificavam diversos padrões de toxicidade dos antimicrobianos: discrasias sanguíneas, anafilaxia, deficiências nutricionais, hipoacusia, convulsões, entre outros. ${ }^{8}$ Estudos recentes corroboram esses achados, apontando a classe de antimicrobianos como importante causadora de reações adversas. ${ }^{11,12}$ Sharma et $\mathrm{al}^{13}$ demonstraram que a classe de antimicrobianos constitui o principal grupo implicado na emergência de reações cutâneas relacionadas à medicamentos, com destaque às sulfonamidas e penicilinas.

A incidência das reações adversas varia entre as diversas publicações, possivelmente devido à ampla gama de drogas envolvidas, dificuldade para a definição de evento adverso ou heterogeneidade das populações avaliadas. Sun et $\mathrm{al}^{14}$ relataram incidência de $24,1 \%$ de eventos adversos em pacientes hospitalizados em uso de antimicrobianos. Valejos ${ }^{15}$ identificou reações leves a moderadas em 55,6\% dos pacientes de uma Unidade de Terapia Intensiva (UTI) pediátrica e neonatal de um hospital universitário de Bogotá.

A presença de reações adversas, está relacionada ao prolongamento da internação hospitalar, com suas consequentes implicações, como o risco de novas infecções, elevação de custos, aumento de morbidade e mortalidade. O já citado artigo de Sun et al demonstrou elevação do tempo de internação 
daqueles pacientes que apresentaram reações adversas quando comparados àqueles livres dos eventos tóxicos. ${ }^{14}$

A diarréia causada por Clostridium difficile (DCD) figura entre uma das mais bem estabelecidas conseqüências indesejáveis do uso de antimicrobianos. Entre os diversos trabalhos da literatura, há variações quanto as principais classes de antimicrobianos implicadas no aparecimento da diarréia. De fato, quase todos os antimicrobianos podem interferir na flora intestinal, eliminando a competição ecológica e favorecendo a multiplicação do C. difficile. ${ }^{16}$ Entretanto, as classes mais comumente relacionadas à incidência de DCD são: cefalosporinas de terceira geração, clindamicina, penicilinas e - mais recentemente - fluoroquinolonas. ${ }^{17,18}$

Há evidências conclusivas de que a incidência de DCD adquirida no hospital está relacionada à intensidade do uso de antimicrobianos no serviço. Estudos recentes demonstraram que a redução de uso de alguns agentes antimicrobianos pode levar a expressivas diminuições na incidência de casos. McNulty et al $^{19}$ demonstraram que a redução no uso de clindamicina diminuiu a incidência de DCD em cerca de 50\%.

Uma revisão sistemática demonstrou evidência significativa de que intervenções sobre a prescrição médica são eficazes para controlar a incidência do $C$. difficile em hospitais. ${ }^{20}$ Essa evidência foi menor no que se referia ao controle da emergência e disseminação de outros microorganismos multirrestistentes. De fato, a relação entre uso de antimicrobianos e resistência é complexa, e merece atenção detalhada. 


\section{Uso de antimicrobianos e resistência.}

A relação entre uso de antimicrobianos e resistência não é tão simples como, a princípio, pode parecer. É um fato reconhecido que existe, ao longo do tempo, uma relação entre a introdução de agentes específicos para uso clínico e, algum tempo depois, o surgimento de resistência em microorganismos originalmente suscetíveis. ${ }^{21}$ No entanto, a relação entre a prevalência de microorganismos resistentes e os padrões de prescrição em determinado hospital é mais difícil de apreender. ${ }^{22}$ Em geral, essa mesma questão é exposta de forma diferente: será possível controlar a disseminação (ou reduzir a incidência) de microorganismos MDR por meio de intervenções sobre a prescrição de antimicrobianos? $?^{20,22,23,24}$ Esta é uma questão central, que procuraremos abordar adiante.

A resistência emerge com mais freqüência em populações com alta incidência de infecções. Além disso, microorganismos MDR disseminam-se com mais eficácia em ambientes propícios a intercâmbios de microbiota. Em locais em que os pacientes permanecem próximos uns aos outros, ocorre facilitação da transmissão cruzada de patógenos entre os pacientes. Todas estas condições estão presentes nas unidades de saúde, particularmente nos hospitais de agudos e, mais ainda, em unidades de terapia intensiva. ${ }^{25}$

Para abordarmos de forma adequada a relação uso/resistência, precisamos nos deter em alguns aspectos. Em primeiro lugar, deve-se compreender de que forma a resistência é relevante para a prática clínica. Os 
microorganismos MDR (definidos como aqueles que apresentam resistência a mais de uma droga rotineiramente utilizada na prática clínica) têm comportamento epidemiológico diverso ${ }^{21}$. Alguns microorganismos, como Acinetobacter ssp pan-resistente ${ }^{26}$ e fungos $^{27}$, acometem grupos específicos, como pacientes em UTIs e imunossuprimidos graves (ex.: pacientes hematológicos). Outros, como o $S$. aureus resistente à meticilina (Methicillinresistant S. aureus, MRSA) apresentam maior disseminação em enfermarias, instituições de longa permanência e mesmo na comunidade. ${ }^{28}$

Entre as diversas espécies de microrganismos de interesse, destacam-se aquelas que, pela alta incidência e relevância clínica, ganharam a denominação de "microrganismos-problema". Este conceito inclui bactérias resistentes a uma ampla gama de antimicrobianos, freqüentemente referidas pela sigla ESKAPE: Enterococcus faecium, S. aureus, Klebsiella pneumoniae, Acinetobacter baumanni, Pseudomonas aeruginosa e Enterobacter ssp. ${ }^{29,30}$

A relação entre uso de antimicrobianos e incidência desses patógenos não segue uma regra única. Partindo de um ponto de vista populacional, Lipsitch \& Samore $^{31}$ propõem alguns modelos através dos quais 0 uso de antimicrobianos influencia a ecologia dos microorganismos e favorece a emergência de cepas resistente. Do ponto de vista individual, os antimicrobianos reduzem a população de isolados sensíveis em um organismo, tornando-o suscetível à aquisição de cepas resistentes. Além disso, a ausência de competição permite a multiplicação de microorganismos resistentes já carreados pelo indivíduo, que passam a ser detectáveis e/ou clinicamente relevantes. Do 
ponto de vista coletivo, o uso dos antimicrobianos aumenta a circulação das cepas resistentes no ambiente (hospital ou comunidade) pondo em risco até mesmo aqueles que não fizeram uso dessas medicações.

De um modo geral, a evidência das relações uso/resistência é suportada por estudos ecológicos ${ }^{31,32}$ e observacionais de base individual (caso-controle e coorte). ${ }^{33,34,35}$ Dificuldades metodológicas dessas abordagens têm sido freqüentemente apontadas, levando ao um maior rigor na interpretação de seus resultados..$^{36,37,38,39}$

Uma evidência mais conclusiva do impacto do uso de antimicrobianos sobre a incidência de microorganismos MDR poderia ser fornecida por estudos de intervenção. Nestes casos, a partir de uma alteração nos padrões de prescrição, espera-se que ocorra redução na prevalência de patógenosproblema MDR no hospital. Vários desses estudos foram incluídos em recentes revisões sistemáticas conduzidas por Ramsay et $\mathrm{al}^{40}$ e Davey et al. ${ }^{20}$

A revisão de Ramsay et $\mathrm{al}^{40}$ deteve-se na qualidade dos dados sobre impacto da intervenção sobre consumo de antimicrobianos. Nesta, de 306 artigos sobre intervenção em práticas de prescrição de antimicrobianos, somente 91 tinham critérios mínimos compatíveis com um protocolo de "prática efetiva e organização de assistência" (effective practice and organization of care, EPOC). Destes, a maioria não apresentava adequadamente dados préintervenção, e vários utilizavam testes estatísticos não apropriados. 
O trabalho de Davey et al $^{20}$ enfrenta a difícil questão de identificar impacto das intervenções sobre a incidência de microorganismos MDR e C. difficile. Como a maioria desses estudos utilizava abordagem estatística inadequada, os autores refizeram a análise de séries temporais. Como anteriormente mencionado, os resultados mais convincentes diziam respeito à redução na incidência de DCD. Em segundo lugar, identificou-se evidência limitada (mas real) de que interenções sobre padrões de prescrição poderiam reduzir a incidência de resistência em bactérias Gram-negativas. Por outro lado, não foi possível afirmar o mesmo para as Gram-positivas. Os autores chamam a atenção para o fato de que, neste último caso, há "falta de evidência", e não "evidência de que a intervenção não tem efeito". Em outras palavras, há necessidade de estudos mais rigorosos para esclarecimento deste assunto. Esse achado demonstra que o uso de antimicrobianos e sua relação com a resistência representam um campo aberto para a pesquisa científica.

\section{Relação entre uso inadequado de antimicrobianos e prognóstico.}

Um dos princípios fundamentais da terapia antimicrobiana é o emprego de agentes de máxima eficácia. A terapia anti-infecciosa sub-ótima tem sido associada a diversos efeitos indesejáveis, como falha terapêutica, aumento do tempo de internação, elevação dos custos hospitalares e até mesmo morte. ${ }^{41,42}$ Este fato é mais significativo em UTIs. Nestas unidades, foi demonstrado grave 
impacto negativo no prognóstico de pneumonias, especialmente aquelas associadas à ventilação mecânica. Estudos sugerem que o erro na escolha do antimicrobiano para instituição imediata pode resultar em elevação da letalidade da ordem de $40 \% .{ }^{43}$ Risco semelhante foi observado em infecções da corrente sangüínea. ${ }^{44}$ Em um estudo de coorte conduzido em uma UTI neonatal, Apisarnthanarak et al observaram mortalidade mais elevada em pacientes com sepse que haviam sido abordados com antimicrobianos de espectro insuficiente. ${ }^{45}$ As graves conseqüências da instituição de antimicrobianos subótimos também foram demonstradas para outras infecções (p.ex.: intraabdominais). ${ }^{46}$

A evidência sugere a necessidade de medidas para otimizar o uso de antimicrobianos, como a utilização de guias terapêuticos baseados em evidência científica, o conhecimento de padrões locais de etiologia e suscetibilidade aos antimicrobianos e da existência de programas institucionais para assegurar o uso racional desses medicamentos. ${ }^{47,48}$

\section{Iniciativas globais para o uso correto dos antimicrobianos.}

Diante do impacto da elevação de prevalência de bactérias multirresistentes e suas consequências, diversas iniciativas foram lançadas por todo o mundo na tentativa de elaborar estratégias para a contenção da disseminação de resistência. ${ }^{41}$ Em 1998 a Organização Mundial de Saúde (OMS) publicou um comunicado "urgente" recomendando a todos os países que mobilizassem 
esforços no sentido de elaborar manuais que regulamentassem o uso de antimicrobianos. ${ }^{49}$ Em 2001, o conselho europeu publicou suas recomendações para o uso prudente de antimicrobianos na medicina humana, que consistiam em: (a) estabelecer ou melhorar os sistemas de vigilância sobre a resistência e sobre o consumo de drogas antimicrobianas; (b) implementar controle e medidas preventivas que estimulassem o uso racional de agentes antimicrobianos; (c) promover educação e treinamento dos profissionais de saúde sobre a resistência microbiana e (d) esclarecer a população quanto à importância do uso racional destes medicamentos. ${ }^{50}$

Medidas como esta demonstram a apreensão das autoridades de saúde pública frente à escalada da incidência de microorganismos MDR principalmente no ambiente hospitalar, mas também na comunidade. Por essa razão, a promoção do uso racional de antimicrobianos tornou-se uma prioridade para diversas organizações (governamentais ou não), entre elas: Organização Mndial da Saúde, Aliança para Uso Prudente de Antimicrobianos, Centros de Prevenção e Controle de Doenças dos Estados Unidos (Centers for Disease Control and Prevention, CDC), Sociedade Americana de Epidemiologia em Serviços de Saúde (Society of Healthcare Epidemiology of America, SHEA) e Sociedade Americana de Doenças Infecciosas (Infectious Diseases Society of America, IDSA). Várias dessas instituições produziram ou endossaram guias práticos para instituição da supervisão de antimicrobianos em hospitais. ${ }^{51}$ 


\section{Programas de supervisão de antimicrobianos em hospitais.}

A constatação de que os antimicrobianos representavam algumas das medicações mais utilizadas no ambiente hospitalar, determinando profundo impacto financeiro, levou ao estabelecimento de medidas administrativas de controle de prescrições já na década de $1970 .^{52}$ Apesar da reconhecida importância de controlar custos da assistência, esse viés prejudicou a abordagem do uso incorreto dos antimicrobianos, freqüentemente interpretada pelo corpo clínico como medida invasiva e restritiva realizada para finalidade unicamente financeira. ${ }^{41}$

Nas últimas duas décadas, políticas com o objetivo de melhorar a prescrição dos agentes anti-infecciosos no hospital sofreram grande mudança conceitual. ${ }^{53}$ Em primeiro lugar, passaram a enfocar a qualidade da atenção, objetivando garantir melhor prognóstico para os pacientes e a redução da emergência e disseminação de microorganismos MDR. ${ }^{54}$ Além disso, passaram a ser vistas como uma das múltiplas medidas de controle da resistência bacteriana, necessariamente aplicada em conjunto com estratégias de adesão à higienização das mãos, instituição de precauções de isolamento e intervenções para redução na incidência de infecções hospitalares. ${ }^{55}$ Nesse contexto, o termo "controle de antimicrobianos" for substituído por "supervisão do uso de antimicrobianos" (tradução aproximada do termo em inglês, antimicrobial stewardship). ${ }^{53,54}$ 
Os programas de supervisão de antimicrobianos (PSAs) receberam importante apoio com a publicação de recomendações práticas da IDSA. Estas recomendações estão contidas em dois documentos: um relacionado à prevenção e controle de resistência ${ }^{56}$ e outro enfocando a estrutura e objetivos dos programas destinados a promover o uso racional dos antimicrobianos. ${ }^{57}$

No documento que aborda os PSAs, ${ }^{57}$ enfatizam-se alguns temas importantes:

- É necessária uma equipe básica, idealmente constituída por médicos infectologistas e farmacêuticos clínicos.

- É fundamental o apoio da administração do hospital.

- As medidas de controle de antimicrobiano devem ser feitas em conjunto com estratégias direcionadas ao controle das infecções hospitalares (ou relacionadas à assistência em saúde).

- Idealmente, o PSA deve ser constituído sob os auspícios de um programa de qualidade e segurança hospitalar.

As estratégias instituídas em PSAs são essencialmente duas:

- Auditoria prospectiva com retorno ao prescritor. Nesse caso, obtém-se diariamente uma lista de pacientes recebendo antimicrobianos, e, a partir dela, sugerem-se ao prescritor as alterações necessárias. A auditoria geralmente não envolve ações restritivas mandatórias, o que apresenta 
vantagens (o prescritor não sente sua autonomia tolhida) e desvantagens (não há como assegurar adesão às intervenções). ${ }^{58,59}$

- Estratégias de pré-autorização, nas quais alguns antimicrobianos somente são liberados após aprovação por um membro do PSA, geralmente acionado por telefone em escala de plantão. ${ }^{60,61}$ Apesar de permitir controle mais intenso sobre a prescrição, esta estratégia tem sua aplicação dificultada por dois problemas. Em primeiro lugar, o rígido controle pode gerar insatisfação na equipe médica. Por outro lado, foram detectadas imprecisões acidentais ${ }^{62,63}$ e mesmo propositais ${ }^{64}$ nas comunicações por telefone.

$\mathrm{Na}$ verdade, vários serviços utilizam estratégias mistas, intermediárias ou complementares. Entre estas, podemos citar: o uso de fichas de solicitação de antimicrobianos com avaliação restritiva posterior, intervenções sobre a disponibilidade de antimicrobianos na farmácia, controle informatizado e publicação de guias terapêuticos locais. ${ }^{53,54}$ Algumas dessas estratégias merecem discussão detalhada.

A ciclagem de antibióticos foi por diversas vezes preconizada como forma a evitar a pressão seletiva de apenas algumas drogas por longo período de tempo - em teoria, diminuindo a chance de emergência de resistência. Essa técnica mostrou-se promissora em estudos iniciais. ${ }^{65}$ Em publicação de 2006, Kollef ${ }^{66}$ avaliou a eficácia da utilização desta prática em ambiente de UTI. Após o levantamento das publicações da época, foi possível inferir que o rodízio de 
antimicrobianos não se provou efetivo quando utilizado isoladamente. O rodízio de antibióticos também foi alvo de uma revisão sistemática, conduzida por Brown \& Nathwani ${ }^{67}$ que concluiu que não existiam estudos suficientes e livres de falhas metodológicas que permitissem a recomendação rotineira desta prática. Por fim, modelos matemáticos e estudos epidemiológicos sugeriram que maior heterogeneidade no uso (ou seja, usar alternativamente vários esquemas diferentes para tratar síndromes infecciosas semelhantes), conhecidos como "mixagem de antibióticos", apresentam maior potencial de impacto sobre a resistência que a estratégia clássica de ciclagem. ${ }^{68,69}$

Programas envolvendo assistência computacional à decisão foram aplicados com sucesso em diversos hospitais. ${ }^{70,71}$ Neles, durante a prescrição (geralmente já informatizada) o médico recebe uma série de informações que podem incluir os antimicrobianos sugeridos para síndromes clínicas específicas, ou dados sobre dosagem e duração de tratamento. Essa abordagem tem sido associada à redução de custos e otimização da terapia.

Resta falar das estratégias educativas, como a produção de guias terapêuticos, complementada por aulas e reuniões com corpo clínico. Embora sejam componentes essenciais de um PSA, estas são certamente as medidas que apresentam menor impacto quando realizadas isoladamente. Está demonstrado que as recomendações são rapidamente esquecidas e a adesão a protocolos, ao longo do tempo, negligenciada. ${ }^{41,51}$ Provavelmente, para que tenham efeito, estratégias educativas devem ser direcionadas a itens 
estratégicos (identificados pelo PSA) e realizadas em conjunto com medidas restritivas.

De um modo geral, a efetividade dos PSAs tem sido relatada em estudos do tipo "antes e depois". Embora estes estudos apresentem algumas limitações, quanto à validade da interpretação de dados e definição de desfechos ${ }^{20,40}$, seus resultados são reconfortantes. Vários deles apresentam redução consistente no consumo de antimicrobianos, custo e incidência de resistência. ${ }^{58,59,60,61,70,71,72,73}$ Em alguns, tomou-se o cuidado de avaliar se a restrição de antimicrobianos havia causado algum dano aos pacientes. Em geral, não havia dano ou mesmo se identificava melhora no prognóstico..$^{41,51}$

Estudos tipo "antes e depois" apresentam uma limitação adicional: somente são aplicáveis para avaliar uma intervenção. Mas existe também a necessidade de avaliações periódicas para identificar necessidades de aprimoramento de PSAs. Tais estudos devem ser delineados de forma a identificar itens que requeiram intervenção e prover um retorno às equipes dos PSAs quanto à efetividade de suas ações. Nesse sentido, estudos que determinam incidência e fatores preditores de erros nas prescrições podem fornecer informações valiosas. 


\section{Estudos de incidência e preditores de erros na prescrição de antimicrobianos.}

Kunin et $\mathrm{al}^{9}$ foram pioneiros no empreendimento de categorizar incorreções na prescrição de antimicrobianos. Em seu artigo clássico 1973, os autores realizaram ampla abordagem do tema, discutindo suas raízes e impactos sobre a prática clínica. Como complemento, relataram os resultados de um levantamento realizado no hospital da Universidade da Virgínia, em

Charlottesville. Por um período de três meses, foram acompanhadas as internações em unidades clínicas (405) e cirúrgicas (390). Ao todo, 27\% dos pacientes das enfermarias clínicas e $29 \%$ daqueles de unidades cirúrgicas receberam antibióticos com finalidade terapêutica. Adicionalmente, antibióticos profiláticos foram administrados a $58 \%$ dos pacientes cirúrgicos e $6 \%$ dos clínicos. Ao todo, $49 \%$ dos antimicrobianos introduzidos foram classificados como apropriados ou provavelmente apropriados. Os demais apresentavam incorreções diversas: possibilidade de escolha de medicação mais barata ou menos tóxica (27\%), erros de posologia (9\%) e mesmo uso desnecessário (17\%). A classificação de incorreções proposta por Kunin et al tornou-se clássica e passou a ser utilizada (com eventuais modificações) por outras publicações.

Outro estudo importante, que aprimorou os critérios de Kunin et al, foi realizado por Jones et $\mathrm{al}^{74}$ no Hospital de Veteranos de Dallas, Texas. Esse 
estudo avaliou o uso de antimicrobianos em dois inquéritos de seis semanas, antes e depois de intervenção educativa para os médicos do serviço. Observouse que o total de prescrições inapropriadas diminuiu de $60 \%$ para $56 \%$. O impacto foi mais significativo na redução de profilaxias cirúrgicas injustificadas (84\% para $55 \%)$.

Outros autores seguiram a trilha de Kunin \& Jones, o que resultou em diversas publicações descrevendo padrões de incorreção na prescrição de antimicrobianos em hospitais de todo o mundo. Tunger et $\mathrm{al}^{75}$ descrevem dois estudos transversais realizados com sete anos de intervalo em um hospital de 300 leitos na Turquia. No primeiro estudo (1998), 54,3\% dos antimicrobianos haviam sido prescritos de forma incorreta (em 12\% dos casos, desnecessária). Entre os dois levantamentos foi montada uma política restritiva para diversas classes de medicamentos anti-infecciosos. No segundo estudo (2005), somente 8,6\% dos antimicrobianos prescritos apresentavam alguma recomendação. Neste grupo, a proporção de casos de tratamento desnecessário era irrisória $(1,5 \%)$. Outros dados do estudo chamam atenção: a proporção de casos tratados com base em exames microbiológicos subiu de 4,7\% para 14,5\%. Erros de prescrição eram mais incidentes em enfermarias cirúrgicas que em unidades clínicas.

Dumo et $\mathrm{al}^{76}$ avaliaram o uso de antimicrobianos em um hospital nas Filipinas, identificando erros em $60,3 \%$ das prescrições de profilaxia e em $45,7 \%$ daquelas com finalidade terapêutica. Somente $6,4 \%$ das prescrições haviam sido realizadas após consulta com médico especialista em doenças infecciosas. 
Um estudo realizado em Porto Alegre (RS) avaliou 3.389 solicitações de antimicrobianos, caracterizando $17,8 \%$ delas como não apropriadas. ${ }^{77}$ Utilizando uma metodologia diferente (seis inquéritos de prevalência de ponto realizados entre 2001 e 2004), Willemsen et al $^{78}$ reportaram que $37,4 \%$ das solicitações de antimicrobianos em um hospital da Holanda eram incorretas. Erbay et $\mathrm{al}^{79}$ identificaram proporção semelhante de erros $(35,8 \%)$ em um hospital na Turquia onde havia um PSA ativo com poderes restritivos. Os mesmos autores realizaram um outro estudo, enfocando apenas o uso de antimicrobianos em UTIs, e observaram incorreção em $47 \%$ das prescrições. ${ }^{80} \mathrm{Na}$ Tailândia, Apisarnthanarak et $\mathrm{al}^{81}$ constataram incorreções em $25 \%$ dos antimicrobianos prescritos.

Uma abordagem diferente - mas igualmente interessante - foi utilizada por Hecker et $a^{82}$ para quantificar o uso desnecessário de antimicrobianos em um hospital de 650 leitos em Cleveland, nos Estados Unidos. Os autores acompanharam por duas semanas as prescrições de antimicrobianos para pacientes internados em unidades não intensivas. A unidade de análise eram os dias de uso dos antimicrobianos, e havia especial preocupação com agentes anaerobicidas. Em 1.941 dias totais de antibioticoterapia, 576 (30\%) eram desnecessários. Os principais motivos para erro eram tratamentos de quadros não infecciosos e de colonização, além de não interrupção de antimicrobianos no momento indicado. O custo total dos dias de tratamento desnecessário foi de US\$14.599,98. 
Entre os estudos citados acima, diversos utilizaram modelos estatísticos uni e multivariados para identificar preditores de uso incorreto. Entre os fatores associados a erros, alguns são dignos de nota: pacientes de unidades cirúrgicas $^{80,81}$ ou obstétricas, prescrição não baseada em estudos microbiológicos ou de antimicrobianos sem restrição de acesso ${ }^{79}$, uso de quinolonas $^{78}$. Por outro lado, a maioria desses estudos identificou a avaliação prévia por médico infectologista como fator protetor contra uso inapropriado de antimicrobianos. $^{79,80,81}$

Via de regra, percebemos duas limitações em estudos que avaliam fatores preditores de incorreção: (1) estes estudos analisam apenas um pequeno número de fatores e (2) as análises dizem respeito a erros em geral, e muitas vezes incluem prescrições com finalidade terapêutica e profilática em uma só categoria. Em nossa opinião, tais generalizações podem prejudicar a validade dos achados. Isso nos levou a desenvolver um estudo com a finalidade de superar essas limitações e fornecer informações para aprimoramento de um PSA.

\section{Controle de Antimicrobianos no Hospital Estadual Bauru}

O Hospital Estadual Bauru (HEB) é um hospital terciário de 285 leitos ativos, inaugurado em 2002. Ele possui quatro UTIs, uma unidade de atendimento de pacientes queimados e diversas enfermarias de pacientes clínicos, cirúrgicos e 
pediátricos. É um hospital de ensino, recebendo alunos do curso médico, residentes e pós-graduandos.

O HEB é administrado por uma fundação ligada à Faculdade de Medicina de Botucatu, Universidade Estadual Paulista. Desde que foi inaugurado, o hospital mantém uma Comissão de Controle de Infecção Hospitalar $(\mathrm{CCIH})$ ativa, e esta desenvolve um PSA educativo/restritivo. Entre as medidas realizadas nesse PSA estão: a instituição de guias terapêuticos locais, reuniões freqüentes com o corpo clínico, possibilidade de avaliação por médico infectologista previamente à prescrição de antimicrobianos e auditoria restritiva (por meio de formulário escrito) de todos os antibióticos de uso parenteral.

Como mencionamos acima, o fato de haver um PSA instituído desde 2002 sem grandes alterações não permite a realização de análises tipo "antes e depois". Por essa razão - e para que fosse possível identificar situações que necessitassem de intervenção - optamos pela realização de estudo de preditores de incorreção, incluindo na o maior número possível de fatores e avaliando em separado as diferentes categorias de erro. 


\section{REFERÊNCIAS DA INTRODUÇÃO}

1. Mendelsohn JA. 'Like all that lives': biology, medicine and bacteria in the age of Pasteur and Koch. Hist Philos Life Sci. 2002; 24: 3-36.

2. Trevett A, Lalloo D. A new look at an old drug: artemesinin and qinghaosu. P N G Med J. 1992; 35: 264-9

3. Hippocrates. On ulcers (translated by Translated by Francis Adams) [online: http://classics.mit.edu//Hippocrates/ulcers.html].

4. Pillai SK, Eliopoulos GM, Moellering Jr RC. Principles of anti-infective therapy. In: MANDELL GL, BENNET JE, DOLIN R. Mande'll, Douglas and Bennett's Principles and Practice of Infecious Diseases, $7^{\text {th }}$ Edition. Philadelphia: Churchill-Livingstone 2010; 267-78.

5. Flemming A. On the antibacterial action of cultures of a penicillin, with special reference to their use in the isolation of $B$. influenzae. $\mathrm{Br} J$ Exper Pathol 1929; 10:226-36.

6. Otten $\mathrm{H}$. Domagk and the development of sulphonamides. J Antimicrob Chemother 1989; 17: 696-96.

7. Jawetz E. Antimicrobial chemotherapy. Annu Rev Microbiol 1956; 10: 85114.

8. Eagle H, Saz AK. Antibiotics. Annu Rev Microbiol 1955;9:173-226.

9. Kunin CM, Tupasi T, Craig WA. Use of antibiotics. A brief exposition of the problem and some tentative solutions. Ann Intern Med1973; 79: 555-60. 
10. Alanis AJ. Resistance to antibiotics: are we in the post-antibiotic era? Arch Med Res. 2005; 36: 697-705.

11. Iannini P, Mandell L, Felmingham J, Patou G, Tillotson GS. Adverse cutaneous reactions and drugs: a focus on antimicrobials. $J$ Chemother 2006; 18: 127-39.

12. Owens RC Jr. QT prolongation with antimicrobial agents: understanding the significance. Drugs 2004; 64: 1091-124.

13. Sharma VK, Sethuraman G., Kumar B. Cutaneous adverse reactions: Clinical Pattern and Causative Agents - A 6 Year Series from Chandigarh, India. J Postgrad Med 2001; 47: 95-9.

14. Sun $Y$, Chen $Y$, Wang $Y$ et al. A Prospective Study of Antimicrobial Related Adverse Drug Reactions in Hospitalized Patientes, J Microbiol Immunol Infect 2008; 41: 151-159.

15. Valejos A. Reaciones adversas por antibióticos en una Unidad de Cuidado Intensivo pediátrico y neo-Natal de Bogotá. Biomédica 2007; 27: 66-75.

16. Hookman P, Barkin JS. Clostridium difficile associated infection, diarrhea and colitis. World J Gastroenterol. 2009; 15: 1554-80.

17. Owens RC, Donskey CJ, Gaynes RP et al. Antimicrobial-Associated risk factors for Clostridium difficile Infection. Clin Infect Dis 2008; 46 (Suppl):S19-31.

18. Pépin J, Saheb N, Coulombe N et al. Emergence of fluoroquinolones as the predominant risk factor for Clostridium difficile-Associated Diarrhea: A 
cohort study during an epidemic in Quebec. Clin Infect Dis 2005; 41: 1254 $-60$.

19. McNulty C, Logan M, Donald IP et al. Successful control of Clostridium difficile infection in an elderly care unit through use of a restrictive antibiotic policy. J Antimicrob Chemother. 1997; 40, 707-11.

20. Davey P, Brown E, Fenelon L et al. Systematic review of antimicrobial drug prescribing in hospitals. Emerg Infect Dis 2006; 12: 211-6.

21. Lin M, Weinstein RA, Hayden MK. Multiply drug-resistant pathogens: epidemiology and control. In: Jarvis WE. Bennett and Brachman's Hospital Infections, $5^{\text {th }}$ Editon. Philadelphia (Lippincott Williams \& Wilkins) 2007; 193-222.

22. Lipsictch M, Bergstrom CT, Levin BR. The epidemiology of antibiotic resistance in hospitals: paradoxes and prescriptions. Proc Nat Acad Sci 2000; 97: 1938-43.

23. McGowan Jr JE, Gerdin DN. Does antibiotic restriction prevent resistance? New Horiz. 1996; 4: 370-6.

24. Davey P, Brown E, Fenelon L, et al. Interventions to improve antibiotic prescribing practices for hospital inpatients. Cochrane Database Syst Rev 2005;4:CD003543.

25. Goldman DA. Strategies to prevent and control the emergence and spread of antimicrobial resistant microorganisms in hospitals. JAMA 1996; 275:234-240. 
26. Abbo A, Navo-Venezia S, Hammer-Muntz $O$ et al. Multidrug-resistant Acinetobacter baumannii. Emerg Infect Dis. 2005; 11: 22-29.

27. Abbas J. Candida krusei fungemia: An escalating serious infection in immunocompromised patients. Arch Intern Med 2000; 160: 2659-64.

28. Kevens KR, Morrison MA, Naddle $\mathrm{J}$ et al. Invasive methicillin-resistant Staphyloccocus aureus in the United States. JAMA 2007; 298: 1763-71.

29. Rice LB. Federal funding for the study of antimicrobial resistance in nosocomial pathogens: no ESKAPE. J Infect Dis 2008; 197: 1079-81.

30. Boucher HW, Talbot GH, Bradley JS et al. Bad Bugs, No Drugs: No ESKAPE! An Update from the Infectious Diseases Society of America. Clin Infect Dis 2009; 48: 1-12.

31. MackKenzie F, Bruce J, Stuelens MJ et al. Antimicrobial drug use and infeciont control practices associated with the prevalence of methicillinresistant Staphylococcus aureus in European hospitals. Clin Microbiol Infect 2007; 13: 269-76.

32. Rogues AM, Amadéo B, Venier AG et al. Relationship between rates of antimicrobial consumption and the incidence of antimicrobial resitance in Staphylococus aureus and Pseudomonas aeruginosa isolates from 47 French hospitals. Infect Control Hosp Epidmeiol 2007; 8: 1389-95.

33. D'Agata EMC, Venkataraman L, DeGirolami $P$ et al. Colonization with broad-spectrum cephalosporin-resistant gram-negative bacilli in intensive care units during a nonoutbreak period: prevalence, risk factors, and rate of infection. Crit Care Med 1999; 27:1090-5. 
34. Graffunder EM, Venezia RA. Risk factors associated with nosocomial methicillin-resistant Staphylococcus aureus (MRSA) infection including previous use of antimicrobials. J Antimicrob Chemother 2002; 49: 9991005.

35. Paramythiotou E, Lucet JC, Timsit JF et al. Acquisition of multidrugresistant Pseudomonas aeruginosa in patients in intensive care units: role of antibiotics with antipseudomonal activity. Clin Infect Dis 2004; 38: 6707.

36. Harris AD, Karchmer TB, Carmeli $Y$ et al. Methodological principles of case-control studies that analyzed risk factors for antibiotic resistance: a systematic review. Clin Infect Dis 2001; 32: 1055—61.

37. Harris AD, Samore $\mathrm{MH}$, Lipsitch $\mathrm{M}$ et al. Control-group selection importance in studies of antimicrobial resistance: examples applied to Pseudomonas aeruginosa, Enterococci, and Escherichia coli. Clin Infect Dis 2002;34:1558-1563.

38. Schwaber MJ, De-Medina T, Carmeli Y. Epidemiological interpretation of antibiotic resistance studies - what are we missing? Nat Rev Microbiol 2004;2: 979-83.

39. Fortaleza CM, Freire MP, Moreira Filho DC et al. Risk Factors for Recovery of Imipenem or Ceftazidime-Resistant Pseudomonas aeruginosa Among Patients Admitted to a Teaching Hospital in Brazil. Infect Control Hosp Epidemiol 2006; 27: 901-6. 
40. Ramsay C, Brown E, Hartman G et al. Room for improvement: a systematic review of the quality of evaluations of interventions to improve hospital antibiotic prescribing. J Antimicrob Chemother 2003; 52: 764-71.

41. Fishman NO, Polk RE. Antimicrobial stewardship. In: In: MANDELL GL, BENNET JE, DOLIN R. Mande'll, Douglas and Bennett's Principles and Practice of Infecious Diseases, $7^{\text {th }}$ Edition. Philadelphia: ChurchillLivingstone 2010; 677-85.

42. Kollef $\mathrm{MH}$. Inadequate antimicrobial treatment: an important determinant of outcome for hospitalized patients. Clin Infect Dis 2000; 31 (suppl 4): S131-8.

43. Kollef $\mathrm{MH}$, Sherman $\mathrm{G}$, Ward $\mathrm{S}$ et al. Inadequate antimicrobial treatment of infections: a risk factor for mortality among critically ill patients. Chest $1999 ; 115: 462-74$.

44. Leibovic L, Shraga I, Ducker M et al. The benefit of appropriate antibiotic treatment in patients with bloodstram infections. J Intern Med 1998; 244:379-86.

45. Apisarnthanarak A, Holzmann-Pagal G, Hamvas A et al. Antibiotic use and the influence of inadequate empiric antibiotic therapy on the outcomes of nosocomial bloodstram infections in a neonatal intensive care unit. Infect Control Hosp Epidemiol 2004; 25: 735-41.

46. Krobot K, Yin D, Zhang Q. Effect of inappropriate initial empiric antibiotic therapy on outcome of patients with community-acquired intra-abdominal 
infections requiring surgery. Eur J Clin Microbiol Infect Dis 2004; 23: 6827.

47. Kollef $\mathrm{MH}$. Optimizing antibiotic therapy in the intensive cate unit setting. Crit Care 2001; 5: 189-96.

48. Patterson DL. The role of antimicrobial management programs in optimizing antibiotic prescribing within hospitals. Clin Infect Dis 2006;42 (Suppl2): S90-5.

49. World Health Organization. Emerging and other communicable diseases: antimicrobial resistance. A51/9 - 10 March 1998 [Online: www.who.int]

50. Anonymous: Council Recommendation of 15 November 2001 on the prudent use of antimicrobial agents in human medicine (2002/77/EC). OJEU 2002; 45: 13-16.

51. Owens JR RC. Antimcrobial stewardship: programmatic efforts to optimize antimicrobial use. In: . In: Jarvis WE. Bennett and Brachman's Hospital Infections, $5^{\text {th }}$ Editon. Philadelphia (Lippincott Williams \& Wilkins) 2007; 179-92.

52. John JF, Fishman NO. Programmatic role of the infectious diseases physician in controlling antimicrobial costs in the hospital. Clin Infect Dis $1997 ; 24: 471-4$.

53. Fishman N. Antimicrobial stewardship. Am J Med. 2006 ; 119(suppl 1): S53-61.

54. MacDougall C, Polk RE. Antimicrobial stewardship programs in health care systems. Clin Microbiol Rev 2005; 18: 638-56. 
55. Weinstein RA. Controlling antimicrobial resistance in hospitals: infection control and the use of antibiotics. Emerg Infect Dis 2001; 188-91.

56. Shlaes DM, Gerding DN, John Jr JF. Society for Healthcare Epidemiology of America and Infectious Diseases Society of America joint committee on the prevention of antimicrobial resistance: guidelines for the prevention of antimicrobial resistance in hospitals. Clin Infect Dis 1997; 25:584-99

57. Dellit TH, Owens RC, McGowan Jr JE, et al. Infectious Diseases Society of America and the Society for Healthcare Epidemiology of America gpuidelines for developing an institutional program to enhance antimicrobial stewardship. Clin Infect Dis 2007; 44: 159-77.

58. Fraser GL, Stogsdill P, Dickens Jr JD. Antibiotic optimization: an evaluation of patient safety and economic outcomes. Arch Intern Med 1997; 57: 1689-94.

59. LaRocco Jr A. Concurrent antibiotic review programs - a role or infectious disease specialists at small community hospitals. Clin Infect Dis 2003; 37: 742-43.

60. Gross R, Morgan AS, Kinky DE et al. Impact of a hospital-based antimicrobial management program on clinical and economic outcomes. Clin Infect Dis 2001; 33: 289-95.

61. White Jr AC, Atmar $\mathrm{RL}$, Wilson $\mathrm{J}$ et al. Effects of requiring prior authorization for selected antimicrobials: expenditures, susceptibilities and clinical outcomes. Clin Infect Dis 1997; 25: 230-9. 
62. Linkin DR, Paris S, Fishman NO et al. Inaccurate communications on telephone calls to an antimicrobial stewardship program. Infect Control Hosp Epidemiol 2006; 27: 1374-81.

63. Linkin DL, Fishman NO, Landis $\mathrm{R}$ et al. Effect of communication errors during calls to an antimicrobial stewardship program. Infect Control Hosp Epidemiol 2007; 28: 1374-81.

64. Calfee DP, Brooks I, Zirk NM et al. A pseudo-outbreak of nosocomial infections associated with the introduction of an antimicrobial stewardship program. Infect Control Hosp Epidemiol 2006; 27: 688-94.

65. Masterton RG. Antibiotic cycling: more than it might seem? J Antimicrob Chemother 2005; 55: 1-5.

66. Kollef $\mathrm{MH}$. Is Antibiotic Cycling the Answer to Preventing the Emergence of Bacterial Resistance in the Intensive Care Unit? Clin Infect Dis 2006;43 (suppl): S82-8.

67. Brown EM, Nathwani D. Antibiotic cycling or rotation: a systematic review of the evidence of efficacy. J Antimicrob. 2005; 55: 6-9.

68. Bergstom CT, Lo M, Lipsitch $M$. Ecological theory suggests that antimicrobial cycling will not reduce antimicrobial resistance in hospitals. Proc Nat Acad Sci USA 2004; 36: 13285-90.

69. Martinez J, Josep-María N, Marco F et al. Comparison of antimicrobial cycling and mixing strategies in two medical intensive care units. Crit Care Med 2006; 34: 329-36. 
70. Evans RS, Pestotnik SL, Classen DC et al. A computer-assisted management program for antibiotics and other anti-infective agents. $\mathrm{N}$ Engl J Med 1998; 338: 232-8.

71. Pestotnik SL. Expert clinical decision support systems to enhance antimicrobial stewardship programs: insights from the society of infectious diseases pharmacists. Pharmacotherapy 2005; 25: 327-52.

72. Apisarnthanarak A, Danchaivijitr S, Khawcharoenporn $T$, et al. Effectiveness of education and antibiotic-control program in a tertiary care hospital in Thailand. Clin Infect Dis 2006; 42: 768-75.

73. $\mathrm{Ng} \mathrm{CK}, \mathrm{Wu}$ TC, Chan WM, et al. Clinical and economic impact of an antibiotics stewardship programme in a regional hospital in Hong Kong. Qual Saf Health Care 2008;17:387-92.

74. Jones SR, Pannell J, Barks J, et al. The effect of an educational program upon hospital antibiotic use. Am J Med Sci 1977; 273: 79-85.

75. Tunger O, Karakaya $\mathrm{Y}$, Cetin B et al. Rational antibiotic use. J Infect Developing Countries 2009; 3: 88-93.

76. Dumo Jr. CC, Natino DF, Pena AC et al. Antimicrobial utilization in a tertiary hospital. Phil J Microbiol Infect Dis 1999; 28: 81-4.

77. Behar $\mathrm{P}$, Wagner MB, Freitas $\mathrm{I}$ et al. Assessing the antimicrobial prescription request process in a teaching hospital in Brazil: regulations and training. Braz J Infect Dis. 2000; 4: 76-85. 
78. Willemsen I,, Groenhuijzen A, Bogaers D, et al. Appropriateness of antimicrobial therapy measured by repeated prevalence surveys. Antimicrob Agents Chemother 2007; 51: 864-7.

79. Erbay A, Colpan A, Bodur $\mathrm{H}$ et al. Evaluation of antibiotic use in a hospital with an antibiotic restriction policy. Int $\mathrm{J}$ Antimicrob Agents 2003; 21: 308312.

80. Erbay $A$, Bodur $H$, Akinci $E$ et al. Evaluation of antibiotic use in intensive care units of a tertiary care hospital in Turkey. J Hosp Infect. 2005; 59: 5361.

81. Apisarnthanarak A, Danchaivijitr S, Bailey TC et al. Inappropriate antibiotic use in a tertiary care center in Thailand: an incidence study and review of experience in Thailand. Infect Control Hosp Epidemiol 2006; 27: 416-20.

82. Hecker MT, Aron DC, Patel NP et al. Unnecessary use of antimicrobials in hospitalized patients. Arch Intern Med 2003; 163:972-8. 


\section{OBJETIVOS}

\section{Objetivos gerais}

- Identificar incidência de incorreção na prescrição de antimicrobianos de uso parenteral para finalidade terapêutica para pacientes internados no Hospital Estadual Bauru.

- Identificar preditores de erro na prescrição desses antimicrobianos.

\section{Objetivos específicos}

- Identificar preditores de prescrição desnecessária de antimicrobianos terapêuticos de uso parenteral para pacientes internados no Hospital Estadual Bauru.

- Identificar preditores de prescrição de antimicrobianos de uso parenteral com espectro terapêutico excessivo para pacientes internados no Hospital Estadual Bauru.

- Identificar preditores de prescrição de antimicrobianos de uso parenteral com espectro terapêutico insuficiente para pacientes internados no Hospital Estadual Bauru. 


\section{ARTIGO}

Fatores preditores de erro na solicitação de antimicrobianos de uso parenteral: estudo em um pequeno hospital de ensino no Brasil.

Autores:

Dr. Gustavo Hideki Kawanami

Prof. Dr. Carlos Magno Castelo Branco Fortaleza

Enviado para a revista Quality and Safety in Health Care

Fator de impacto 2.55 (Qualis A2 - Medicina 2) 


\section{RESUMO}

Introdução: O uso inapropriado de antimicrobianos tem impacto negativo sobre o resultado final da internação dos pacientes e sobre a emergência de microrganismos resistentes. A identificação de padrões de prescrição auxilia a melhora do programa de supervisão de antimicrobianos (PSA).

Objetivos: (1) Avaliar a incidência de incorreção em solicitações de antimicrobianos parenterais (SAPs) para finalidade terapêutica em um pequeno hospital de ensino do Brasil. (2) Estudar os fatores preditores de inadequação das SAPs.

Métodos: Um estudo transversal incluiu 25\% das SAPs do ano de 2005. Foram excluídas as prescrições de antimicrobianos profiláticos. Todas as SAPs foram avaliadas independentemente por cada um dos autores. A adequação foi avaliada conforme recomendações dos consensos para antibioticoterapia vigentes naquele ano. A incorreção foi avaliada segundo a classificação de Kunin \& Jones modificada. Em uma segunda etapa, a incorreção foi avaliada através de modelos uni e multivariados. Todas as SAPs classificadas como "Apropriadas" ou "Provavelmente Apropriadas" foram selecionados como controles. Os grupos de "casos" foram compostos pelas SAPs com incorreções (gerais ou específicas).

Resultados: Foram avaliadas 963 prescrições, das quais 34,6\% foram consideradas "inapropriadas". Os fatores preditores gerais de incorreção foram: Prescrição nos finais de semana e feriados $(O R=1,67, I C 95 \%=1,20-1,28$, 
$p=0,002)$, pacientes em unidade de terapia intensiva $(O R=1,57, I C 95 \%=1,11$ 2,23, $p=0,01)$, infecção peritoneal $(O R=2,15, I C 95 \%=1,27-3,65, p=0,004)$ ou infecção em trato urinário $(\mathrm{OR}=1,89, \mathrm{IC} 95 \%=1,25-2,87, \mathrm{p}=0,002)$, combinação de dois ou mais antimicrobianos (OR=1,72, IC95\%=1,15-2,57, $p=0,008)$, prescrição de uma droga da classe das penicilinas $(\mathrm{OR}=2,17,95 \% \mathrm{Cl}=1,38-3,41$, $p=0,001)$ ou de cefalosporina de primeira geração $(O R=1,74, I C 95 \%=1,01-3,00$, $p=0,048)$. A consulta prévia ao infectologista foi protetora contra o uso inapropriado $(\mathrm{OR}=0,34, \mathrm{IC} 95 \%=0,24-0,50, \mathrm{p}<0,001) . \quad$ Fatores independentes associados a erros específicos variaram, mas a cons,ulta prévia ao infectologista foi protetora contra uso desnecessário $(\mathrm{OR}=0,04, \mathrm{IC} 95 \%=0,01-0,26, \mathrm{p}=0,001)$ e prescrição de antimicrobianos de espectro insuficiente (OR=0,14, IC95\%=003$0,30, p=0,01)$

Conclusão: $O$ estudo demonstra a importância da consulta prévia ao infectologista em assegurar a qualidade das prescrições de antimicrobianos. Ele também indica os padrões de prescrição que devem ser abordados pelas políticas do PSA. 


\section{INTRODUÇÃO}

Apesar dos benefícios da terapia anti-infecciosa serem inquestionáveis, o uso inapropriado dos agentes antimicrobianos pode ter impactos negativos sobre a qualidade de atenção à saúde. ${ }^{1} \mathrm{O}$ uso excessivo de antimicrobianos está associado a eventos adversos graves ${ }^{2,3}$, diarréia associada a Clostridium difficile $^{4}$ e à emergência e disseminação de microrganismos multirresistentes. ${ }^{5-7}$ Por outro lado, a terapia antimicrobiana insuficiente pode levar à falha de tratamento, prolongamento do tempo de hospitalização e em último caso, à morte. ${ }^{8}$ Além disso, o uso inapropriado tem impactos diretos e indiretos sobre os custos hospitalares. ${ }^{9,10}$

Para garantir qualidade no uso dos antimicrobianos, muitos hospitais desenvolveram programas de supervisão de antimicrobianos (PSAs). ${ }^{11,12}$ Estes programas geralmente compreendem medidas educativas, a possibilidade de consultas ao especialista em doenças infecciosas, políticas de pré-autorização ou auditoria prospectiva dos padrões de prescrição. Apesar de haver diversos relatos dos benefícios do controle de antimicrobianos em ambientes hospitalares ${ }^{13-15}$, os PSAs devem manter subsídios para seu contínuo desenvolvimento.

A identificação dos padrões de prescrição inapropriadas pode auxiliar na identificação dos fatores que necessitam de intervenções dos PSAs. Este estudo pretende identificar fatores preditores de prescrições incorretas em um pequeno hospital de ensino com um ativo PSA. Apesar dos custos hospitalares serem 
dados relevantes, o estudo foi conduzido de forma a evitar o viés financeiro, com a análise realizada somente sobre dados clínicos.

\section{MÉTODOS}

\section{Local do Estudo}

O estudo foi conduzido no Hospital Estadual Bauru (HEB), instituição afiliada à Faculdade de Medicina de Botucatu. O HEB possui 285 leitos e está localizado na cidade de Bauru, estado de São Paulo, Brasil. O hospital provê atendimentos secundários e terciários a uma área de aproximadamente um milhão de habitantes. Possui quatro unidades de terapia intensiva, uma unidade de queimados e várias enfermarias que recebem pacientes clínicos, cirúrgicos e pediátricos. Exerce também papel de hospital de ensino para estudantes de medicina, internos e residentes.

O hospital possui laboratório de microbiologia e uma ativa Comissão de Controle de Infecções Hospitalares (CCIH). A CCIH mantém um PSA educativo/restritivo desde a inauguração do hospital em 2002. Durante o ano de realização deste estudo (2005) a CCIH/PSA estava composta por três infectologistas.

\section{Política do PSA}

A política do PSA deste hospital compreende intervenções pré e pós prescrição:

(1) Emissão de manuais terapêuticos locais, periodicamente atualizados e 
apresentados em reuniões do corpo clínico; (2) possibilidade de consulta ao infectologista antes da prescrição; (3) obrigatoriedade do preenchimento da ficha de solicitação de antimicrobianos para drogas parenterais (e algumas drogas orais). Estas solicitações são auditadas pela equipe de CCIH/PSA no dia seguinte, com a possibilidade de restrição ou alteração da prescrição do antimicrobiano.

\section{Desenho do Estudo}

Foi realizado estudo transversal, com a finalidade de a avaliar os fatores preditores de inadequação nas solicitações de antimicrobianos parenterais (SAPs) para uso terapêutico do ano de 2005 . Um total de $25 \%$ das solicitações daquele ano foram aleatoriamente selecionadas para a análise. Foram excluídas as solicitações de antimicrobianos orais, assim como as SAPs com propósito profilático.

\section{Identificação e classificação de inadequação}

Os dados foram colhidos à partir de SAPs e suplementados com informações do prontuário e arquivos do laboratório. Todos estes dados foram agrupados para análise de acordo com as "Instâncias de Prescrição" (IPs). Uma IP foi definida como a solicitação de um ou mais antimicrobianos, com ao menos 48 horas de intervalo a partir da prescrição de quaisquer outras drogas antimicrobianas prévias para o mesmo paciente. Cada uma das IPs foi submetida à avaliação de adequação pelos autores. Quando possível, a 
adequação foi julgada baseando-se nas orientações terapêuticas da Sociedade Americana de Doenças Infecciosas (Infectious Diseases Society of America, IDSA) vigentes no ano do estudo. ${ }^{16-24}$ Nos casos para os quais estas recomendações não estavam disponíveis, o parecer de adequação foi emitido à partir de outras evidências científicas e/ou padrões de resistência antimicrobiana locais. Foi empregada uma versão modificada dos clássicos critérios de Kunin \& Jones $^{25,26}$ para classificar o uso de antimicrobianos. As categorias definidas foram:

Apropriada (A): Prescrição de antimicrobiano baseada na correta interpretação de resultados microbiológicos confiáveis, ou em pleno acordo com publicações de manuais terapêuticos baseados em evidência.

Provavelmente Apropriada (PA): Prescrição de base empírica, não expressa nas recomendações da IDSA, porém utilizando antimicrobiano(s) de espectro e farmacocinética/farmacodinâmica adequados para 0 tratamento daquela infecção.

Desnecessária (D): Prescrição de antimicrobiano(s) para paciente sem infecção ou com infecção já corretamente tratada.

Espectro Excessivo ou Redundante (E/R): Prescrição de antimicrobiano de espectro excessivamente amplo ou prescrição de associação de drogas em número maior que o necessário.

Espectro Insuficiente (I): Prescrição de antimicrobiano cujo espectro não inclui os agentes conhecidos ou prováveis para a infecção presente. 
Duração curta ou Dose baixa (C/B): O tempo de tratamento ou a dose solicitada é insuficiente para se atingir resposta terapêutica.

Duração Longa ou Dose Alta (L/A): O tempo de tratamento ou a dose é mais elevada que o necessário para atingir resposta terapêutica.

Múltiplos Erros (M): Qualquer combinação das categorias D, E/R, I, C/B ou L/A. Para diminuir fatores subjetivos, cada solicitação de IP foi analisada independentemente pelos dois autores. A concordância entre os autores foi medida através do coeficiente de kappa. ${ }^{27}$ Para casos discordantes, foi realizada uma re-classificação consensual.

\section{Análise de Fatores Preditores}

Para a análise, foram coletados dados adicionais relacionados aos pacientes: características demográficas, data da internação, uso prévio de antimicrobiano e unidade da internação. Outros dados relevantes colhidos foram: data da prescrição, consulta prévia ao infectologista e a utilização de resultados microbiológicos na justificativa da SAP. Foram selecionados 4 desfechos de interesse: inadequação em geral e erros específicos (D, E/R e I). Cada um dos desfechos foi avaliado em análises individuais. Em todos os desfechos, as categorias A e PA foram utilizadas como controles.

\section{Análise estatística}

Todos os dados foram submetidos à análise univariada. O teste exato de Fisher e Chi-quadrado foram utilizados para variáveis dicotômicas. Variáveis 
numéricas foram analisadas através do teste $T$ de Student ou $U$ de MannWhitney.

A seleção das variáveis para a análise multivariada (regressão logística) foi realizada utilizando-se o processo Stepwise Forward ("change-in-estimate method'). ${ }^{28} \mathrm{Em}$ resumo, as variáveis com $\mathrm{p}<0.2$ na análise univariada foram incluídas em modelo de regressão logística. Aquelas estatisticamente significantes $(p<0.05)$ nesta análise, foram colocadas em outro modelo. Os novos modelos incluíram (uma-a-uma) todas as variáveis do estudo, sendo testadas para colinearidade e confusão. Confundidores foram mantidos no modelo final, quando eram capazes de alterar o Odds Ratio (OR) de qualquer uma das variáveis significantes em mais de $10 \%$.

\section{RESULTADOS}

No ano do estudo (2005), 11.529 pacientes foram admitidos no Hospital Estadual Bauru. Destes, 28,7\% receberam ao menos um antimicrobiano parenteral durante a internação, 64,2\% com propósito terapêutico. Um número total de 1.501 solicitações de antimicrobianos foram selecionados para a análise. Excluindo-se os antimicrobianos orais e as SAPs para uso profilático, foram identificados 963 IPs. As IPs foram analisadas independentemente pelos dois autores e classificadas em categorias de prescrição. O valor do kappa para concordância entre os avaliadores foi de $0.89(p<0,001)$. 
De todas as prescrições analisadas, $42,7 \%$ foram realizadas nas primeiras 48 horas de admissão, $24,8 \%$ em finais de semana ou feriados. Foram identificadas combinações de duas ou mais drogas antimicrobianas em $17,3 \%$ das IPs. Em 21,0\% a prescrição havia sido realizada em leito de terapia intensiva, em $32,6 \%$ o prescritor presumiu haver infecção relacionada aos cuidados de saúde e em 6,9\% alegou-se o embasamento em resultados microbiológicos na justificativa da prescrição.

Foi detectada incorreção em $34,6 \%$ de todas as IPs. As categorias de prescrição estão listadas na tabela 1. O PSA realizou intervenções pósprescrição em $77,5 \%$ daquelas incorretas e em $22,5 \%$ daquelas classificadas como A ou PA em nossa análise $(p<0,001)$. 
Tabela 1. Classificação das solicitações de antimicrobianos de uso parenteral conforme os critérios de Kunin \& Jones modificados. ${ }^{25,26}$

\begin{tabular}{lcc}
\hline Categoria & Instâncias de Prescrição & $\%$ \\
\hline Apropriado (A) & 480 & 49.8 \\
Provavelmente Apropriado (PA) & 150 & 15.6 \\
Desnecessário (D) & 74 & 7.7 \\
Excessivo/Espectro Redundante (E/R) & 59 & 6.1 \\
Espectro Insuficiente (I) & 45 & 4.7 \\
Curta Duração ou Dose baixa (C/B) & 45 & 4.7 \\
Duração Longa ou dose alta (L/A) & 99 & 10.3 \\
Múltiplos erros (M) & 11 & 1.1 \\
\hline
\end{tabular}

Preditores de inadequação conforme resultados da análise univariada estão mostrados nas tabelas 2 e 3 . A tabela 4 mostra os resultados da análise multivariada. 


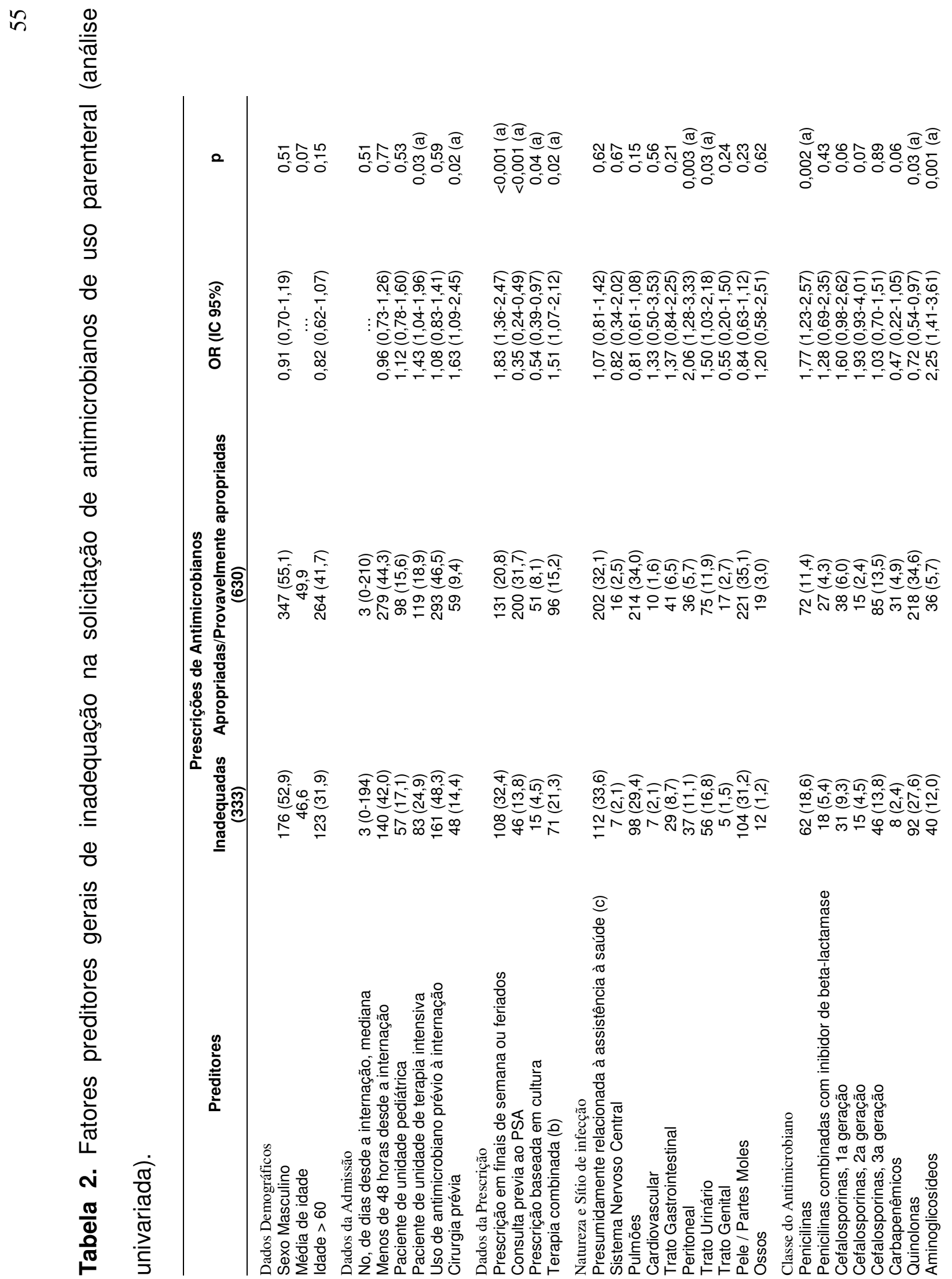


in

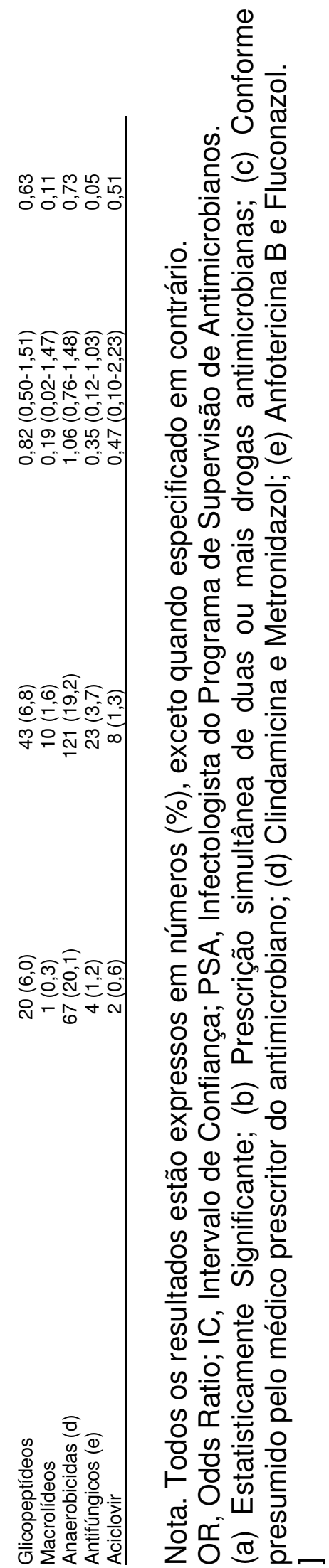




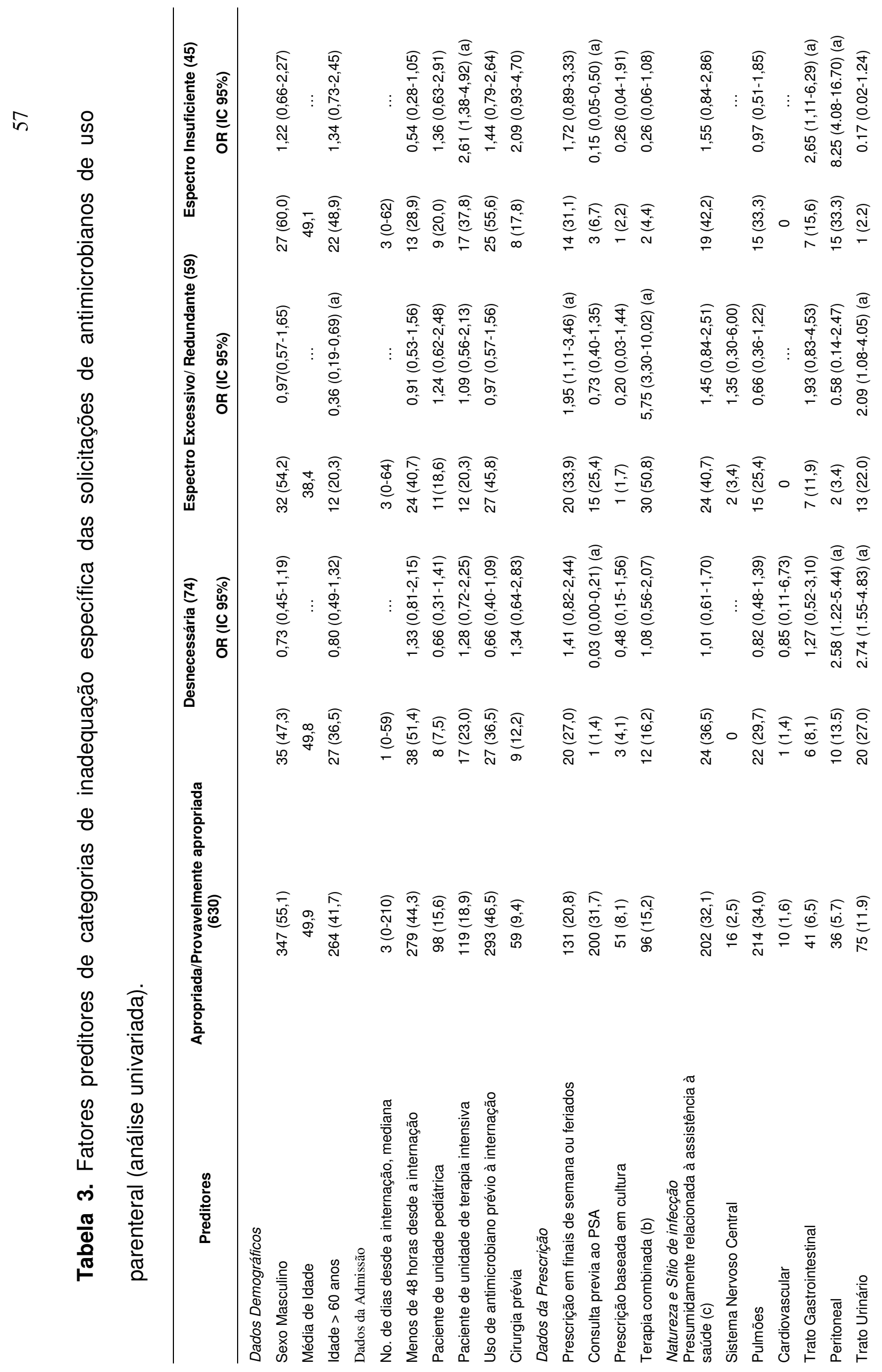




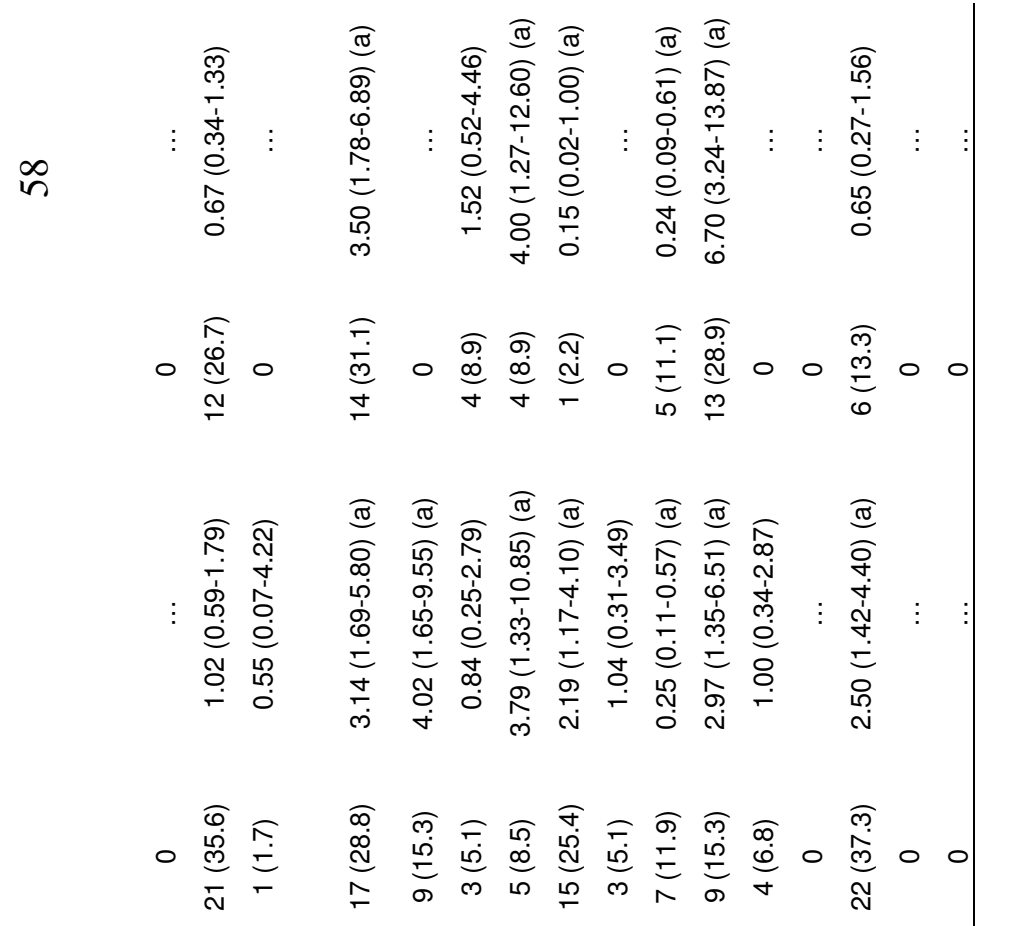

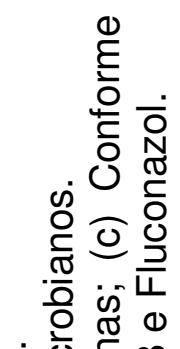

으음

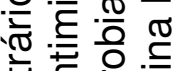

紊这

ठ० 0.

ฮิ

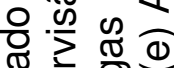

잉

응 응

की

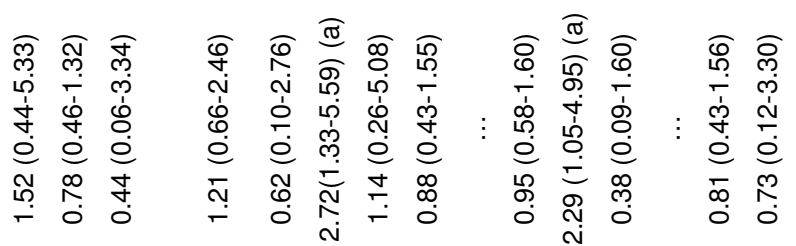

๙ $\varepsilon$

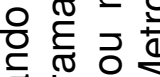

宁

은 은 ำ

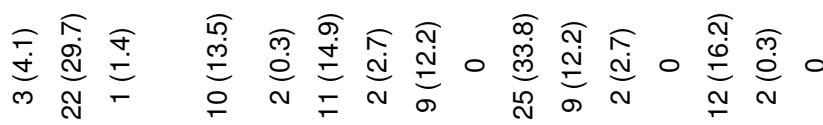

엉 응 ชิ

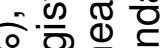

응 空

웡 吾

ब

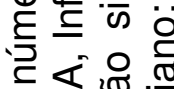

ह

(1) U

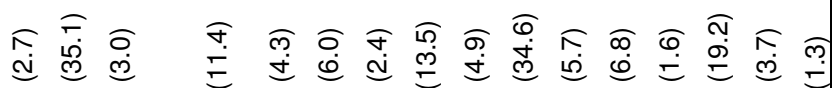

ऽ 엉

की $\frac{\bar{\sigma}}{\square}$

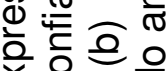

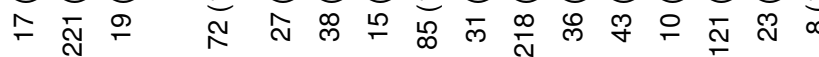

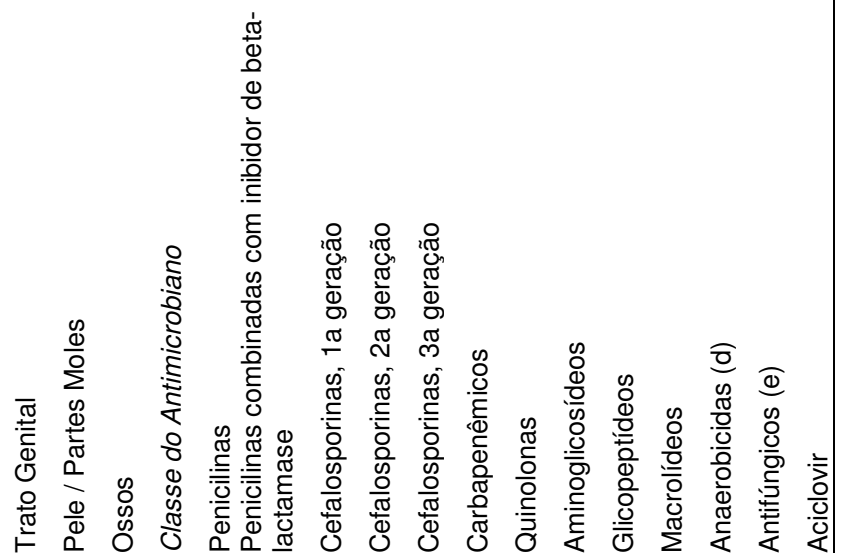

बU

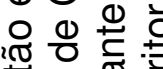

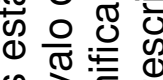

क \ 등

융 㐫

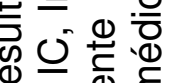

○纪

이ㅇㅡㅠ 응 응

요믐

응 옹 옹응

응 흥

뉴웛 
Tabela 4. Fatores preditores de inadequação nas solicitações de antimicrobianos de uso parenteral (análise multivariada).

\begin{tabular}{lcc}
\hline \multicolumn{1}{c}{ Preditores } & OR (IC95\%) & p \\
\hline Prescrição Inadequada (geral) & $1.57(1.11-2.223)$ & 0.01 \\
Paciente em Terapia Intensiva & $1.67(1.20-2.28)$ & 0.002 \\
Prescrição em Finais de semana/Feriados & $0.34(0.24-0.50)$ & $<0.001$ \\
Consulta prévia ao PSA & $2.15(1.27-3.65)$ & 0.004 \\
Infecção Peritoneal & $1.89(1.25-2.87)$ & 0.01 \\
Infecção de Trato Urinário & $2.12(1.39-3.25)$ & 0.001 \\
Droga da classe das penicilinas & $1.74(1.01-3.00)$ & 0.048 \\
Cefalosporinas, 1a geração & $1.72(1.15-2.57)$ & 0.008 \\
Terapia combinada (a) & & \\
Uso de Antimicrobiano Desnecessário (D) & $2.77(1.51-5.11)$ & 0.001 \\
Infecção de Trato Urinário & $4.31(1.85-10.07)$ & 0.001 \\
Infecção Peritoneal & $2.28(1.05-4.91)$ & 0.04 \\
Cefalosporinas, 1a geração & $0.04(0.01-0.26)$ & 0.001 \\
Consulta prévia ao PSA & & \\
Espectro Excessivo / Redundante & $0.39(0.18-0.84)$ & 0.02 \\
Idade > 60 anos & $2.73(1.34-5.55)$ & 0.006 \\
Prescrição em Finais de semana/Feriados & $22.54(7.78-65.30)$ & $<0.001$ \\
Terapia combinada & $8.22(3.05-22.5)$ & $<0.001$ \\
Infecção de Trato Urinário & $0.65(0.02-0.16)$ & $<0.001$ \\
Quinolonas & $0.16(0.04-0.63)$ & 0.008 \\
Aminoglicosídeos & & \\
Espectro Insuficiente & $8.34(2.55-27.31)$ & $<0.001$ \\
Paciente em Terapia Intensiva & $0.14(0.03-0.3)$ & 0.01 \\
Consulta prévia ao PSA & $91.15(17.88-464.63)$ & $<0.001$ \\
Infecção Peritoneal & $0.04(0.00-0.54)$ & 0.02 \\
Infecção de Trato Urinário & & \\
\hline
\end{tabular}


Os fatores independentemente associados à inadequação foram a prescrição em finais de semana ou feriados, pacientes admitidos nas Unidades de Terapia Intensiva (UTIs), infecções peritoneais ou de trato urinário, a combinação de duas ou mais drogas antimicrobianas e a terapia com penicilina ou cefalosporinas de primeira geração. A consulta prévia ao infectologista foi protetora contra a prescrição inadequada.

A prescrições desnecessárias (categoria “D”) ocorreram principalmente quando o objetivo da prescrição era o tratamento de infecções peritoneais ou do trato urinário, ou quando a prescrição envolvia o uso de cefalosporinas de primeira geração. Por outro lado, a chance de incorrer neste padrão de incorreção se mostrou diminuída quando havia consulta prévia ao infectologista.

Prescrições realizadas nos finais de semana e feriados, para tratamento de infecções do trato urinário ou em associações de antimicrobianos, foram fatores de risco independentes para a categoria "E/R". Este erro foi menos provável naqueles pacientes com 60 anos ou mais ou na prescrição de quinolonas ou aminoglicosídeos.

A admissão em UTI e as infecções peritoneais foram fatores preditores independentes para as prescrições da categoria "l". Os fatores protetores foram a infecção localizada no trato urinário e a consulta prévia ao infectologista. 


\section{DISCUSSÃO}

Nas últimas duas décadas, as políticas hospitalares de controle de antimicrobianos mudaram suas medidas predominantemente relacionadas à contenção de custos para estratégias de promoção da qualidade. ${ }^{29} \mathrm{~A}$ tentativa de melhorar o uso de antimicrobianos e evitar resistência levou ao desenvolvimento do PSA do Hospital Estadual Bauru desde sua inauguração. Isto nos deixou uma herança paradoxal: tornou-se difícil avaliar o impacto do PSA através de análises de "antes e depois". ${ }^{13,30}$ Este estudo tentou superar o problema realizando uma análise exaustiva dos fatores relacionados às prescrições inadequadas.

A proporção de prescrições de antimicrobianos inapropriados em nosso hospital foi similar àquelas descritas em outros estudos. ${ }^{31,32}$ Entretanto, 0 presente trabalho se diferencia destes e dos demais estudos em diversas maneiras. Em primeiro lugar, foram excluídos da análise todos os antimicrobianos prescritos com finalidade profilática. Em nossa opinião, as particularidades do uso de antimicrobianos profiláticos merecem ser avaliadas em estudos específicos. ${ }^{37}$

Apesar de a avaliação de fatores preditores de incorreção geral ter sido realizada de forma similar às publicações anteriores ${ }^{31-35}$, este estudo refinou sua análise abordando erros específicos (categorias "D", "E/R" e "I"). Entre os resultados encontrados, foi possível notar que a generalização pode ser enganosa. Quando consideramos as infecções de trato urinário, é perceptível 
que pacientes com este diagnóstico apresentaram chance elevada de receberem tratamento com antibióticos de espectro excessivamente amplo ou mesmo desnecessário. De fato, em muitos destes pacientes havia apenas bacteriúria assintomática, sem necessidade de tratamento. De maneira oposta, porém coerente, um diagnóstico de infecção de trato urinário foi protetor contra a instituição de terapia com antimicrobiano de espectro insuficiente. Este é um breve exemplo de detalhes que poderiam ter sido perdidos em uma análise de "erros em geral".

A presença de peritonite também foi associada um padrão duplo de incorreção. Essa infecção foi frequentemente diagnosticada em excesso, porém, quando estava presente, era tratada com antimicrobianos insuficientes. Foi possível detectar um erro considerado grave para um hospital de ensino: a falta da associação de uma droga anaerobicida às quinolonas ou aminoglicosídeos.

Outros achados também são dignos de nota. As cefalosporinas de primeira geração estavam frequentemente associadas às presumidas (porém inexistentes!) infecções de pele, sítio cirúrgico e trato urinário. Erros referentes à prescrição de penicilinas foram encontrados em várias categorias. Por vezes as prescrições que continham penicilina foram classificadas na categoria "l" (penicilina ou oxacilina para tratamento de pneumonias comunitárias, por exemplo), porém por outras vezes foi encontrada como parte de uma combinação "E/R" (oxacilina associada a cefepime para pneumonia adquirida na comunidade ou oxacilina associada a aminoglicosídeo e metronidazol para tratamento de peritonite). Aminoglicosídeos e quinolonas, quando não faziam 
parte de uma associação, foram mais comumente prescritos com indicação correta. Por fim, foi encontrado um dado preocupante: pacientes de UTIs foram mais comumente tratados com antimicrobianos de espectro insuficiente.

O achado mais notável deste estudo foi a importância do especialista em doenças infecciosas em prevenir prescrições inadequadas: seja para erros em geral, ou para as categorias "D" ou "l”. Apesar de se mostrarem já tão significantes, estes achados ainda podem estar subestimados. De fato, muitas das consultas ao infectologista deste estudo foram realizadas por telefone e não puderam ser quantificadas em nosso estudo. Evidências indiretas mostram um benefício ainda maior das consultas prévias ao infectologista. As prescrições realizadas em finais de semana ou feriados (quando não havia especialistas em doenças infecciosas disponíveis para consulta) estavam comumente incluídas na categoria "E/R". A clara importância da consulta ao infectologista já foi extensamente demonstrada em estudos prévios realizados em países desenvolvidos ${ }^{38}$ e em desenvolvimento. ${ }^{32,34,35}$ Este é um forte argumento para a importância universal do PSA como um instrumento para a promoção da qualidade da assistência à saúde.

Alguns aspectos deste estudo merecem explicação. Em primeiro lugar, não foram analisados os preditores de erros classificados nas categorias " $C / B$ " $\mathrm{e}$ "L/A". Como o estudo foi baseado em "solicitações", que foram comumente alteradas pela equipe do PSA, os dados sobre doses e duração refletiam, na melhor da hipóteses, a intenção daquele que realizou a prescrição. Estes dados 
não foram considerados consistentes o suficiente para merecer análise específica.

Pode-se acreditar que a categorização do uso de antimicrobianos pelos autores tenha sido afetada por fatores subjetivos. Por este motivo, as análises foram realizadas de forma independente e utilizou-se o coeficiente de kappa para se medir a concordância entre os avaliadores. Os valores de kappa medem o quanto a concordância excede aquela que é esperada pelo acaso. O coeficiente de kappa deste estudo (0.89) é classificado como "concodância quase completa" segundo a classificação de Landis \& Koch. ${ }^{39}$

Diversas mudanças foram instituídas no PSA deste hospital após este estudo: (1) Possibilidade de consulta do especialista em doenças infecciosas nos finais de semana e feriados; (2) Visitas diárias dos membros do PSA às UTIs; (3) solicitação informatizada ("online") de antimicrobianos; e (4) comunicação direta dos resultados microbiológicos aos membros do PSA. Apesar de novos estudos serem necessários para a quantificação do impacto destas medidas, analises preliminares sugerem o que a proporção de prescrições baseadas em resultados de culturas aumentou em pelo menos duas vezes.

Concluindo, a análise dos fatores preditores de inadequação nas prescrições de antimicrobianos permitiu a identificação de elementos que necessitavam intervenções. O estudo também forneceu informações sobre a eficácia do PSA, demonstrando a importância da consulta prévia ao especialista 
em doenças infecciosas para assegurar a qualidade das prescrições antimicrobianas.

\section{REFERÊNCIAS BIBLIOGRÁFICAS}

1. van der Meer JW, Gyssens IC. Quality of antimicrobial drug prescription in hospital. Clin Microbiol Infect 2001; 7 (suppl 6):12-5.

2. Iannini P, Mandell L, Felmingham J, Patou G, Tillotson GS. Adverse cutaneous reactions and drugs: a focus on antimicrobials. J Chemother 2006;18:127-39.

3. Owens RC Jr. QT prolongation with antimicrobial agents: understanding the significance. Drugs 2004;64:1091-124.

4. Hookman $P$, Barkin JS. Clostridium difficile associated infection, diarrhea and colitis. World J Gastroenterol. 2009;15:1554-80.

5. Goldmann DA, Weinstein RA, Wenzel RP et al. Strategies to Prevent and Control the Emergence and Spread of Antimicrobial-Resistant Microorganisms in Hospitals. A challenge to hospital leadership. JAMA. 1996; 275:234-40.

6. Safdar N, Maki DG. The commonality of risk factors for nosocomial colonization and infection with antimicrobial-resistant Staphylococcus aureus, enterococcus, gram-negative bacilli, Clostridium difficile, and Candida. Ann Intern Med 2002;136:834-44. 
7. Tacconelli E. Antimicrobial use: risk driver of multidrug resistant microorganisms in healthcare settings. Curr Opin Infect Dis. 2009;22:3528.

8. Kollef $\mathrm{MH}$. Inadequate antimicrobial treatment: an important determinant of outcome for hospitalized patients. Clin Infect Dis 2000; 31 (suppl 4):S131-8.

9. Ruttimann S, Keck B, Hartmeier $\mathrm{C}$ et al. Long-term antibiotic cost savings from a comprehensive intervention program in a medical department of a university-affiliated teaching hospital. Clin Infect Dis 2004; 38: 348-56.

10. Sipahi OR. Economics of antibiotic resistance. Expert Rev Anti Infect Ther 2008;6:523-39.

11. Fishman N. Antimicrobial stewardship. Am J Med. 2006 ;119(suppl 1):S53-61.

12. Dellit TH, Owens RC, McGowan Jr JE, et al. Infectious Diseases Society of America and the Society for Healthcare Epidemiology of America guidelines for developing an institutional program to enhance antimicrobial stewardship. Clin Infect Dis 2007; 44:159-77.

13. Ng CK, Wu TC, Chan WM, et al. Clinical and economic impact of an antibiotics stewardship programme in a regional hospital in Hong Kong. Qual Saf Health Care 2008;17:387-92.

14. Martin C, Ofotokun I, Rapp R, et al. Results of an antimicrobial control program at a university hospital. Am J Health Syst Pharm 2005;62:732-8. 
15. Davey P, Brown E, Fenelon L, et al. Interventions to improve antibiotic prescribing practices for hospital inpatients. Cochrane Database Syst Rev 2005;4:CD003543.

16. Tunkel AR, Hartman BJ, Kaplan SL, et al. Practice guidelines for the management of bacterial meningitis. Clin Infect Dis 2004;39:1267-84.

17. Mandell LA, Bartlett JG, Dowell SF, et al. Update of practice guidelines for the management of community-acquired pneumonia in immunocompetent adults. Clin Infect Dis 2003; 2003; 37:1405-33.

18. American Thoracic Society, Infectious Diseases Society of America. Guidelines for the management of adults with hospital-acquired, ventilatorassociated, and healthcare-associated pneumonia. Am J Respir Crit Care Med 2005;171:388-416.

19. Guerrant RL, Van Gilder T, Steiner TS, et al. Practice guidelines for the management of infectious diarrhea. Clin Infect Dis. 2001;32:331-51.

20. Solomkin JS, Mazuski JE, Baron EJ et al. Guidelines for the selection of anti-infective agents for complicated intra-abdominal infections. Clin Infect Dis 2003;37:997-1005.

21. Warren JW, Abrutyn E, Hebel JR, et al. Guidelines for antimicrobial treatment of uncomplicated acute bacterial cystitis and acute pyelonephritis in women. Infectious Diseases Society of America (IDSA). Clin Infect Dis1999;29:745-58. 
22. Nicolle LE, Bradley S, Colgan R, et al. Infectious Diseases Society of America guidelines for the diagnosis and treatment of asymptomatic bacteriuria in adults. Clin Infect Dis 2005;40:643-54.

23. Stevens DL, Bisno AL, Chambers HF, et al. Practice guidelines for the diagnosis and management of skin and soft-tissue infections. Clin Infect Dis $2005 ; 41: 1373-406$.

24. Lipsky BA, Berendt AR, Deery HG, et al. Diagnosis and Treatment of diabetic foot infections. Clin Infect Dis 2004; 39:885-910.

25. Kunin CM, Tupasi T, Craig WA. Use of antibiotics. A brief exposition of the problem and some tentative solutions. Ann Intern Med1973;79:555-60.

26. Jones SR, Pannell J, Barks J, et al. The effect of an educational program upon hospital antibiotic use. Am J Med Sci 1977;273:79-85.

27. Roberts C. Modelling patterns of agreement for nominal scales. Stat Med 2008;27:810-30.

28. Greenland S. Modeling and variable selection in epidemiologic analysis. Am J Public Health 1989;79:340-9.

29. MacDougall C, Polk RE. Antimicrobial stewardship programs in health care systems. Clin Microbiol Rev 2005;18:638-56.

30. Apisarnthanarak A, Danchaivijitr S, Khawcharoenporn T, et al. Effectiveness of education and antibiotic-control program in a tertiary care hospital in Thailand. Clin Infect Dis 2006; 42: 768-75. 
31. Willemsen I,, Groenhuijzen A, Bogaers D, et al. Appropriateness of antimicrobial therapy measured by repeated prevalence surveys. Antimicrob Agents Chemother 2007;51:864-7.

32. Erbay A, Colpan A, Bodur $\mathrm{H}$, et al. Evaluation of antibiotic use in a hospital with an antibiotic restriction policy. Int $\mathrm{J}$ Antimicrob Agents 2003;21:308312.

33. Tünger $O$, Dinç $G$, Özbakkaloglu $B$, et al. Evaluation of rational antibiotic use. Int J Antimicrob Agents 2000;15:131-5.

34. Erbay A, Bodur $\mathrm{H}$, Akinci $\mathrm{E}$, et al. Evaluation of antibiotic use in intensive care units of a tertiary care hospital in Turkey. J Hosp Infect. 2005;59:5361.

35. Apisarnthanarak A, Danchaivijitr S, Bailey TC, et al. Inappropriate antibiotic use in a tertiary care center in Thailand: an incidence study and review of experience in Thailand. Infect Control Hosp Epidemiol 2006;27:416-20.

36. Hecker MT, Aron DC, Patel NP, et al. Unnecessary use of antimicrobials in hospitalized patients. Arch Intern Med 2003;163:972-8.

37. Tan JA, Naik VN, Lingard L. Exploring obstacles to proper timing of prophylactic antibiotics for surgical site infections. Qual Saf Health Care. 2006;15:32-8.

38. Raineri E, Pan A, Mondello P, et al. Role of the infectious diseases specialist consultant on the appropriateness of antimicrobial therapy prescription in an intensive care unit. Am J Infect Control 2008;36:283-90. 
39. Landis JR, Koch GG. The measurement of observer agreement for categorical data. Biometrics 1977;33:159-74. 


\section{CONCLUSÃO}

- Em 963 solicitações de antimicrobianos parenterais para uso terapêutico, $34,6 \%$ apresentavam alguma incorreção.

- Preditores gerais de incorreção foram: prescrição em fins de semana ou feriados, pacientes de Unidades de Terapia Intensiva, peritonite ou infecção urinária, combinação de dois ou mais antimicrobanos e prescrições incluindo penicilinas ou cefalosporinas de primeira geração.

- Peritonites, infecções urinárias e uso de cefalosporinas de primeira geração foram preditores para solicitação desnecessária de antimicrobianos.

- Preditores para solicitação de antimicrobianos com espectro excessivo ou redundante foram: prescrições de finais de semana ou feriados, para tratamento de infecções do trato urinário ou em associações de antimicrobianos. Este erro foi menor em pacientes com mais de 60 anos e em prescrições de quinolonas ou aminoglicosídeos.

- A admissão em UTI e as peritonites foram preditores independentes para as prescrições de antimicrobianos com espectro insuficiente. Infecções do trato urinário estavam associadas a menor risco desse erro.

- A consulta prévia a um médico infectologista foi fator protetor contra prescrição incorreta de antimicrobianos em geral, e especificamente contra a indicação desnecessária e uso de agentes com espectro insuficiente. 


\section{APÊNDICE}

Versão em língua inglesa do artigo enviado para publicação.

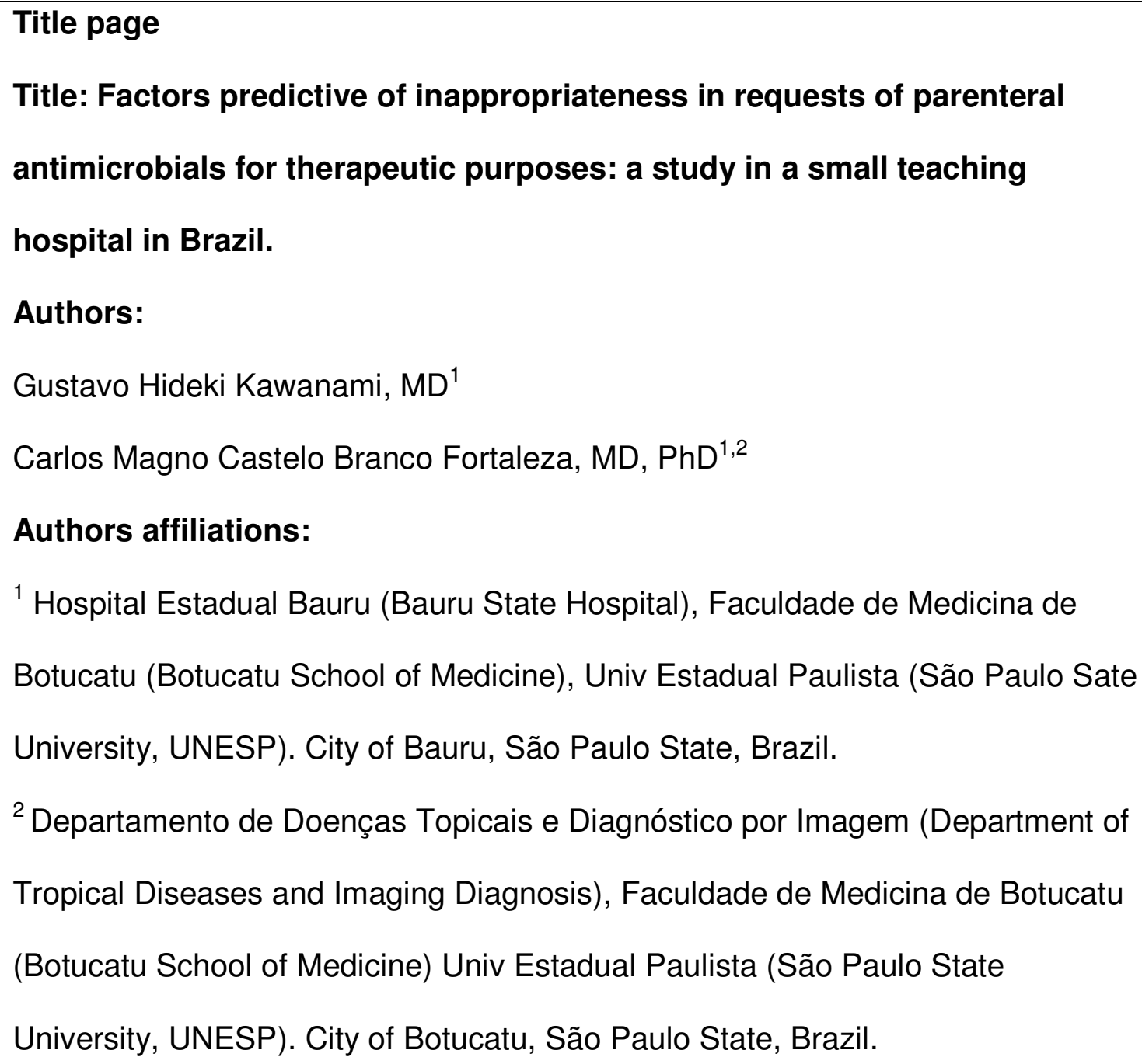




\section{ABSTRACT}

Background: The inappropriate antimicrobial use in hospitals has negative impact in patients' outcomes and is associated with the emergence of drugresistant microorganisms. The identification of patterns of inappropriateness in antimicrobial prescriptions contributes to the improvement of antimicrobial stewardship programs (ASP).

Objective: to identify factors predictive of inappropriateness in requests of parenteral antimicrobials (RPAs) in small teaching hospital.

Methods: A cross-sectional study was conducted. We reviewed $25 \%$ of RPAs for therapeutic purposes from year 2005. Appropriateness was evaluated according to current guidelines for antimicrobial therapy. We assessed predictors of innapropriateness through univariate and multivariable models. All RPAs classified as "appropriate" or "probably appropriate" were selected as controls. "Case" groups comprised inappropriate RPAs (either in general or for specific errors).

Results: Nine hundred and sixty-three RPAs were evaluated, $34.6 \%$ of which were considered inappropriate. General predictors of inapropriateness were: prescription on weekends/holidays $(\mathrm{OR}=1.67,95 \% \mathrm{Cl}=1.20-2.28, \quad \mathrm{p}=0.002)$, patient from Intensive Care Unit $(\mathrm{OR}=1.57,95 \% \mathrm{Cl}=1.11-2.23, \mathrm{p}=0.01)$, peritoneal $(\mathrm{OR}=2.15,95 \% \mathrm{Cl}=1.27-3.65, \mathrm{p}=0.004)$ or urinary tract infection $(\mathrm{OR}=1.89$, $95 \% \mathrm{Cl}=1.25-2.87, \mathrm{p}=0.002)$, combination therapy with two or more antimicrobials $(\mathrm{OR}=1.72,95 \% \mathrm{Cl}=1.15-2.57, \mathrm{p}=0.008)$ and prescriptions including penicillins $(\mathrm{OR}=2.12, \quad 95 \% \mathrm{Cl}=1.39-3.25, \quad \mathrm{p}=0.001)$ or first-generation cephalosporins 
$(\mathrm{OR}=1.74,95 \% \mathrm{Cl}=1.01-3.00, \mathrm{p}=0.048)$. Previous consultation with an infectious diseases (ID) specialist had a protective effect against inappropriate prescription $(\mathrm{OR}=0.34,95 \%=0.24-0.50, \mathrm{p}<0.001)$. Factors independently associated with specific prescription errors varied. However, consultation with ID specialist was protective against both unnecessary antimicrobial use $(\mathrm{OR}=0.04,95 \% \mathrm{Cl}=0.01$ 0.26, $p=0.001)$ and requests of agents with insufficient spectrum $(O R=0.14$, $95 \% \mathrm{Cl}=0.03-0.30, \mathrm{p}=0.01)$.

Conclusion: The study demonstrates the importance of previous consultation with an ID specialist in assuring the quality of antimicrobial prescriptions. Also, it highlights prescription patterns that should be approached by ASP policies. 


\section{INTRODUCTION}

Even though the benefits of anti-infective therapy for hospitalized patients are unquestionable, the inappropriate use of antimicrobial agents adversely impacts the quality of health care. ${ }^{1}$ The overuse of antimicrobials is associated with severe adverse events ${ }^{2,3}$, Clostridium-difficile associated diarrhea ${ }^{4}$ and with the emergence of multidrug-resistant microorganisms..$^{5-7}$ On the other hand, suboptimal antimicrobial therapy may lead to treatment failure, increased length of hospitalization and, ultimately, death. ${ }^{8}$ Additionally, the misuse of antimicrobials in hospitals directly and indirectly impacts on hospital costs. ${ }^{9,10}$

In order to assure the quality of antimicrobial use, many hospitals have developed antimicrobial stewardship programs (ASPs). ${ }^{11,12}$ Those programs comprise education measures, the possibility of consultation with an Infectious Disease (ID) specialist, preauthorization policies and/or prospective audit of prescription patterns. Though there are several reports of the benefits of controlling antimicrobial use in hospital settings ${ }^{13-15}$, ASPs must develop a basis for continual improvement.

The identification of patterns of inappropriate antimicrobial prescriptions can aid in the identification of issues requiring ASP interventions. The present study aims to identify factors predictive of inappropriate antimicrobials requests in a small teaching hospital with an active ASP. Even though costs are an important issue, we attempted to avoid any cost-related bias, and performed the analysis on clinical grounds. 


\section{METHODS}

\section{Setting}

The study was conducted in Hospital Estadual Bauru (Bauru State Hospital), a 285-bed facility affiliated to the Faculdade de Medicina de Botucatu (Botucatu School of Medicine), in São Paulo State, Brazil. The hospital has 285 active beds and provides secondary and tertiary care for a area with approximately one million inhabitants. The infection control committee runs an educative/restrictive ASP since the hospital was opened (in 2002). In the study year (2005), the ICC/ASP team comprised three ID specialists. The ASP policy included the requirement of fulfillment of a request form for every prescription of a parenteral antimicrobial. The requests were audited by the ICC/ASP team in the following day, with the possibility of restricting or changing the prescription.

\section{Study design}

We conducted a cross-seccional study to evaluate factors predictive of inappropriateness in requests for parenteral antimicrobials (RPAs) for therapeuctical purposes in the year 2005. A total of $25 \%$ of requests from that year were randomly selected for analysis. We excluded all requests of oral antimicrobials, as well as RPAs for prophylactic purposes.

\section{Identification and classification of inappropriateness}

Data were collected from the RPAs, supplemented with information from medical charts and grouped in our units for analysis, namely, "instances of prescription" (IPs). An IP was defined as a request of one or more antimicrobials at least 48 hours apart from any other antimicrobial prescription for the same patient. Each 
and every IP was submitted to analysis of appropriateness by the authors. Whenever feasible, appropriateness was judged on the basis of compliance Infectious Diseases Society of America guidelines (IDSA) that were current in the study year. ${ }^{16-24}$ In cases that were not approached by such guidelines, judgment was based on other evidence-based sources. We used a modification of the classical Kunin \& Jones ${ }^{25,26}$ criteria for categories of antimicrobial use. The categories were defined as follows:

Appropriate (A): antimicrobial prescription based on proper interpretation of reliable microbiological results, or fully compliant with published, evidence-based guidelines.

Probably appropriate (PA): prescription on an empirical basis, not matching an IDSA recommendation, but using antimicrobial(s) with spectrum and pharmacokinetic/pharmacodynamic patterns probably adequate to treat the current infection.

Unnecessary $(\mathrm{U})$ : prescription of antimicrobial(s) for a patient without infection, or with a current infection that has been adequately treated.

Excessive or redundant $(E / R)$ spectrum: prescription of antimicrobial(s) with overly broad spectrum, or combination prescription with a greater number of agents that would actually be necessary.

Insufficient (I) spectrum: prescription of antimicrobial agents which spectrum did not include known or probable agents of the current infection.

Short duration or low dosing $(\mathrm{S} / \mathrm{L})$ : the suggested duration or dosing of antimicrobial use is too low to achieve therapeutic response. 
Long duration or high dosing $(\mathrm{L} / \mathrm{H})$ : the suggested duration or dosing of antimicrobial use exceeds the necessary patterns to achieve therapeutic response.

Multiple errors (M): any combination of categories $U, E / R, I, S / L$ and/or $L / H$. In order to minimize subjectivity, IP forms were analyzed independently by the two authors. Inter-rater agreement was measured using kappa coefficient. $^{27} \mathrm{~A}$ consensual reclassification was provided for discordances.

\section{Analysis of predictive factors}

For this analysis, we collected additional information regarding the patient (demographics, admission data), the infection site and the prescription (timing, antimicrobial classes included). Our study selected four outcomes of interest: general inappropriateness, and specific errors of special interest $(U, E / R$, and I categories). Each outcome was assessed in a separate analysis. In all cases, categories $\mathrm{A}$ and PA were used as controls.

\section{Statistical analysis}

Statistical analysis was performed in SPSS version 15.0 (@ SPCC inc). All data were submitted to univariate analysis. Chi-square and Fisher's exact tests were used for dichotomous variables. Numeric variables were analyzed using Student's T test or the Mann-Whitney $U$ test. Variable selection for multivariable analysis (logistic regression) was performed using a stepwise forward process ("change-in-estimate method"). ${ }^{28}$ Variables for which $P<0.2$ in univariate analysis were included in a logistic regression model. Those that were statistically significant $(P<0.05)$ in this analysis were entered in new models, including (one 
by one) all the other study variables. Confounders were kept in the final model whenever they changed the Odds Ratio (OR) of any statistically significant variable by more than $10 \%$.

\section{RESULTS}

In the study year (2005), 11,529 patients were admitted to Hospital Estadual Bauru. Of these, $28.7 \%$ received at least one parenteral antimicrobial during admission $-64.2 \%$ for therapeutic purposes. A total number of 1,501 antimicrobial requests were selected for analysis. Excluding prescriptions of oral antimicrobials and RPAs for prophylactic purposes, we identified 963 IPs. The IPs were analyzed independently by the two researchers and classified into one of the prescription categories. The kappa value for inter-rater agreement was $0.89(p<0.001)$

Inappropriateness was detected in $34.6 \%$ of all IPs. The categories of prescription are listed in table 1. Post-prescription interventions had been performed by ASP in $77.5 \%$ of all inappropriate prescription and in $22.5 \%$ of those that were classified as A or PA in our analysis $(p<0.001)$.

Results from univariate analysis of predictors of inappropriate antimicrobial prescriptions are shown in table 2 and 3. Table 4 depicts results from multivariable analysis. 
Table 1. Classification of parenteral antimicrobial requests according do the modified Kunin \& Jones categories. ${ }^{25,26}$

\begin{tabular}{lcc}
\hline Category & Instances of Prescription & $\%$ \\
\hline Appropriate (A) & 480 & 49.8 \\
Probably appropriate (PA) & 150 & 15.6 \\
Unnecessary (U) & 74 & 7.7 \\
Excessive/redundant spectum (E/R) & 59 & 6.1 \\
Insufficient spectrum (I) & 45 & 4.7 \\
Short duration or low dosing (S/L) & 45 & 4.7 \\
Long duration or high dosing (L/H) & 99 & 10.3 \\
Multiple errors (M) & 11 & 1.1 \\
\hline
\end{tabular}




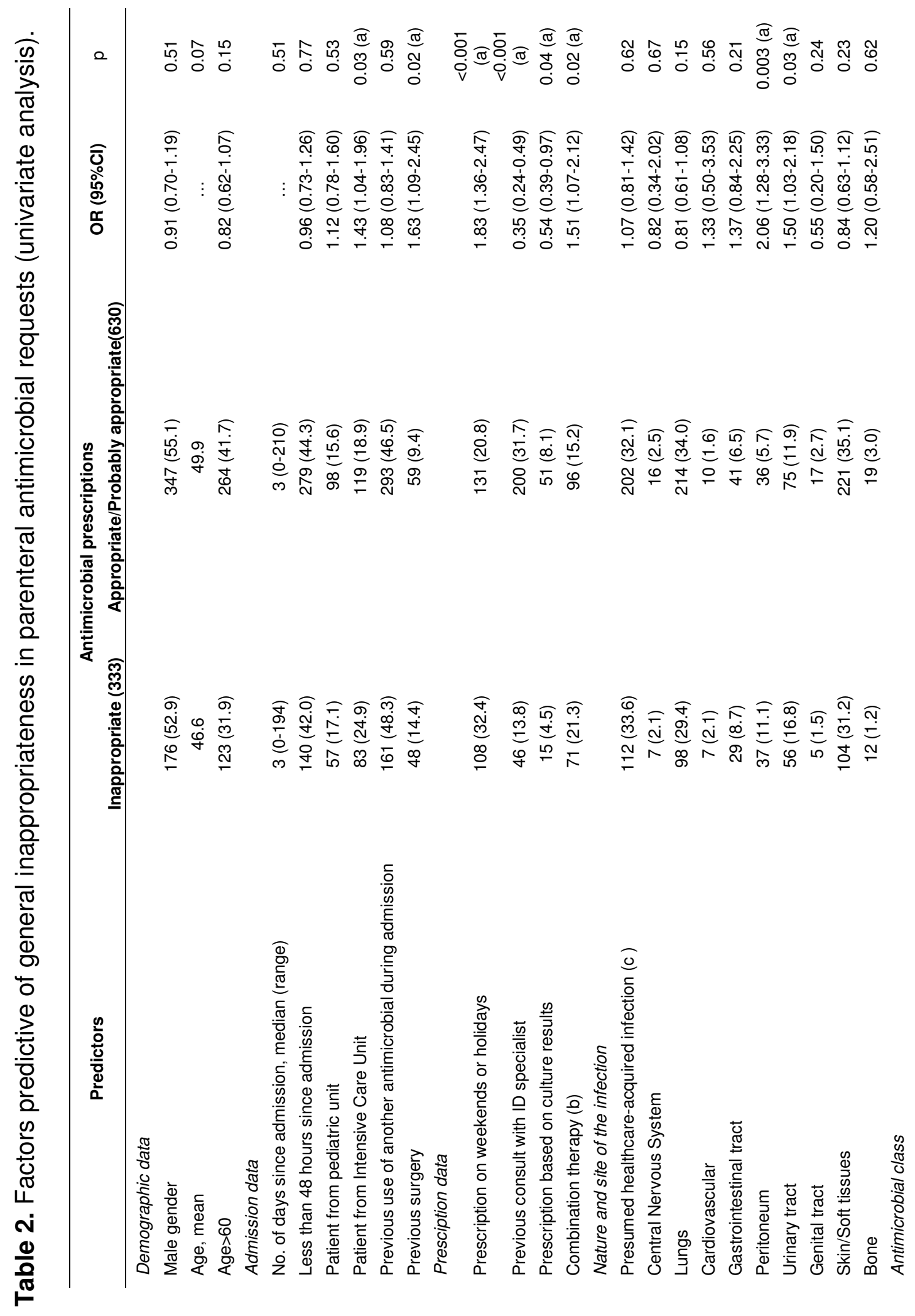




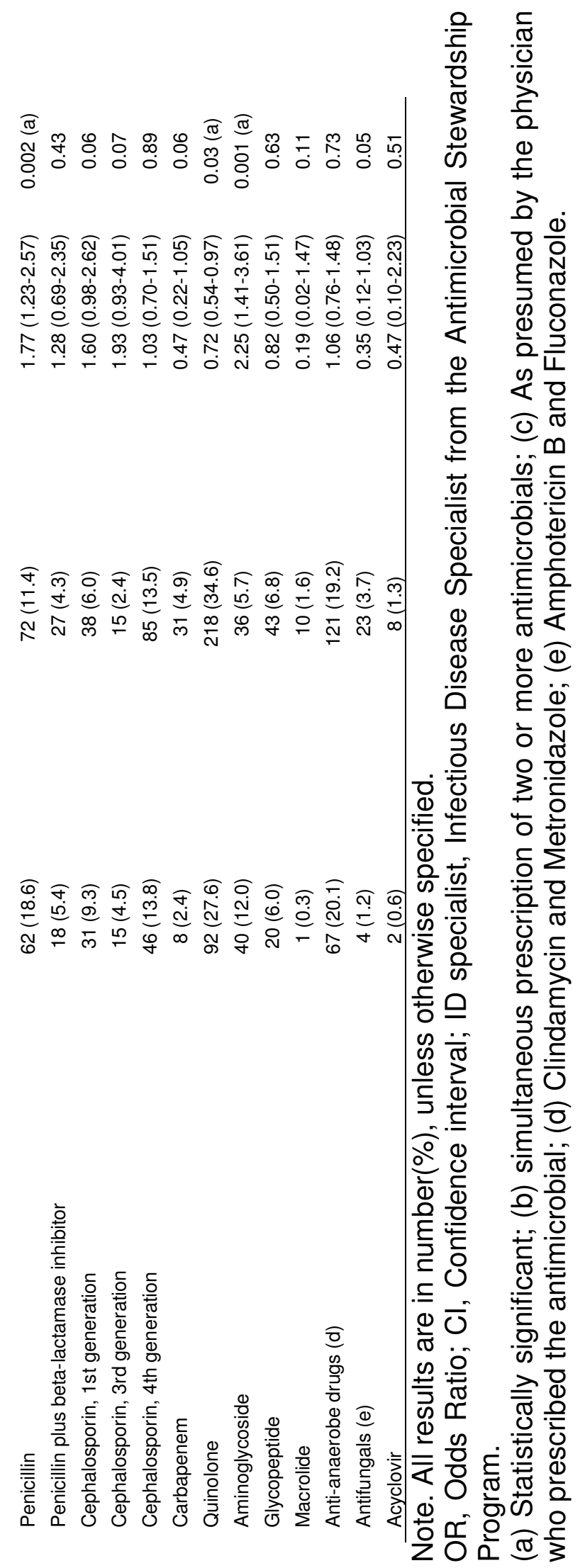




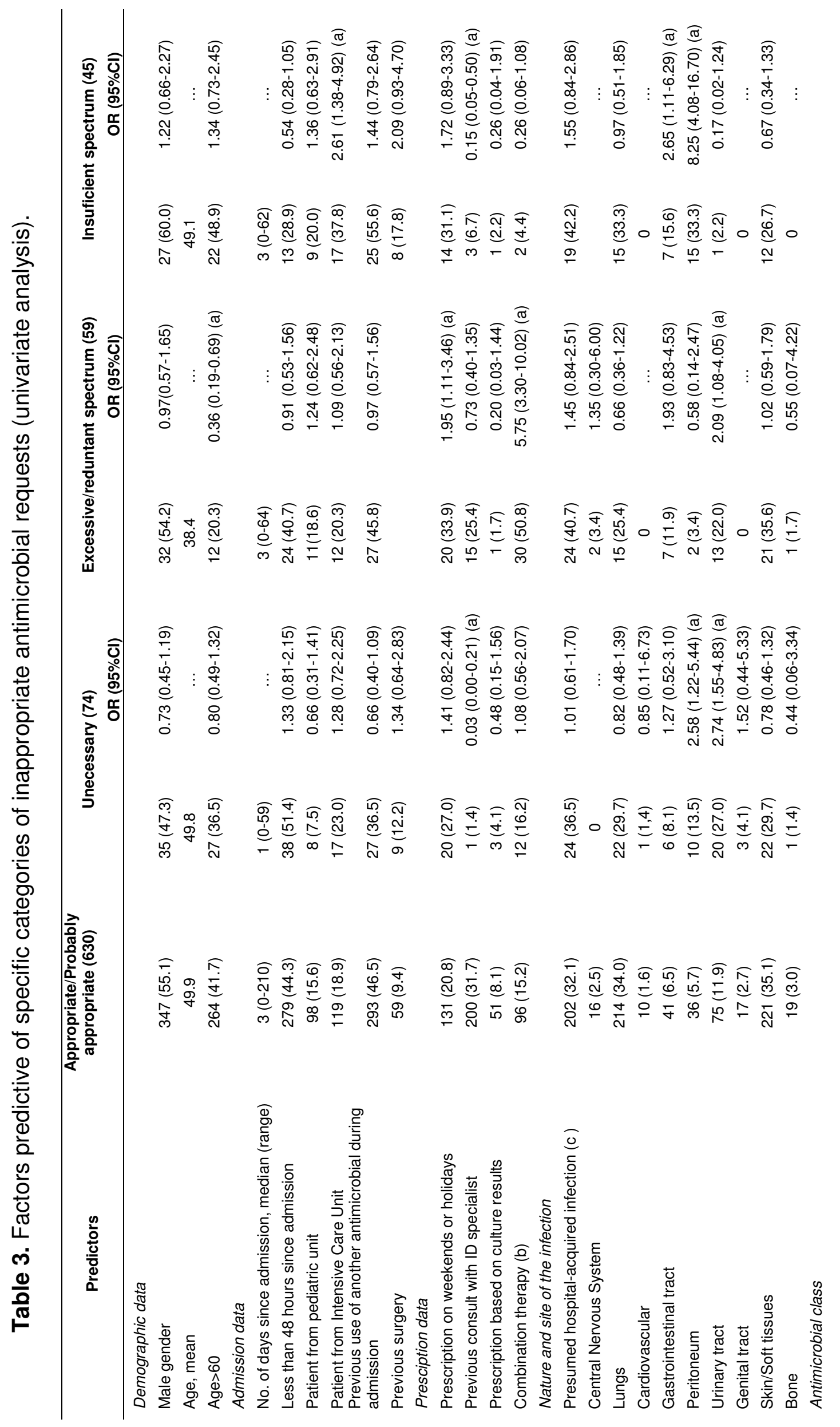




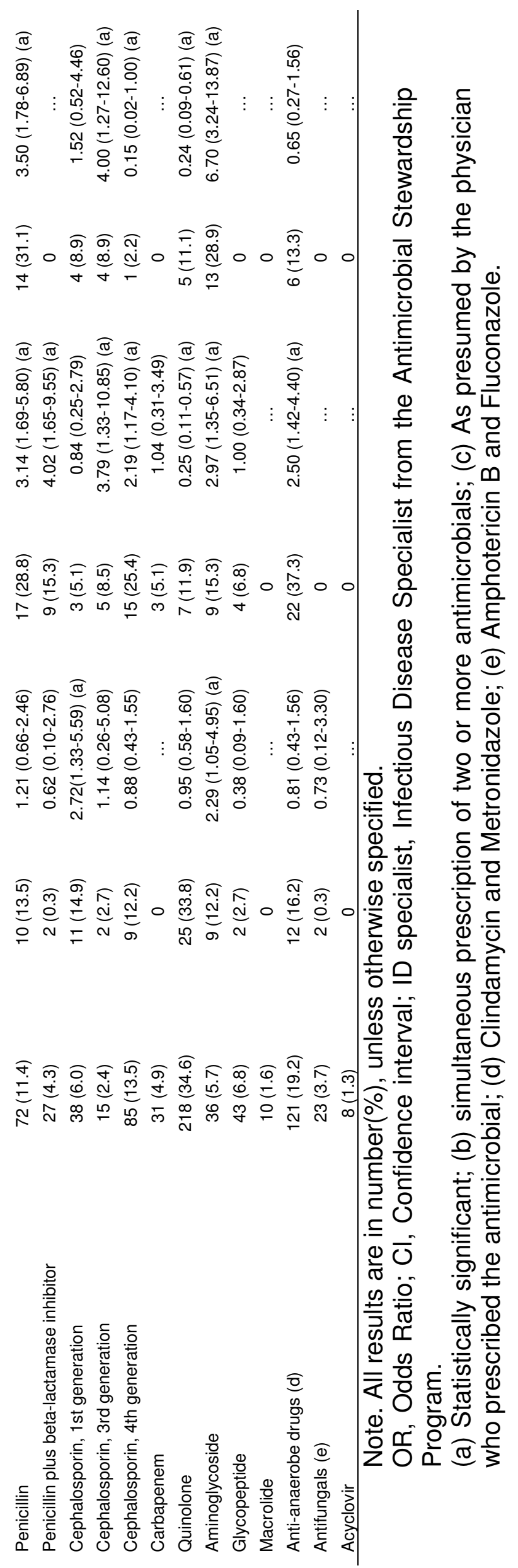


Table 4. Factors predictive of inappropriateness in antimicrobial requests (multivariable analysis).

\begin{tabular}{lcc}
\hline \multicolumn{1}{c}{ Predictors } & OR (95\%Cl) & P \\
\hline Inappropriate prescription (in general) & $1.57(1.11-2.223)$ & 0.01 \\
Patient from Intensive Care Unit & $1.67(1.20-2.28)$ & 0.002 \\
Prescription on weekends/holidays & $0.34(0.24-0.50)$ & $<0.001$ \\
Previous consultation with ID specialist & $2.15(1.27-3.65)$ & 0.004 \\
Peritoneal infection & $1.89(1.25-2.87)$ & 0.01 \\
Urinary tract infection & $2.12(1.39-3.25)$ & 0.001 \\
Drug from penicillin class & $1.74(1.01-3.00)$ & 0.048 \\
Cephalosporin, 1st generation & $1.72(1.15-2.57)$ & 0.008 \\
Combination therapy (a) & & \\
Unnecessary antimicrobial use & $2.77(1.51-5.11)$ & 0.001 \\
Urinary tract infection & $4.31(1.85-10.07)$ & 0.001 \\
Peritoneal infection & $2.28(1.05-4.91)$ & 0.04 \\
Cephalosporin, 1st generation & $0.04(0.01-0.26)$ & 0.001 \\
Previous consult with ID specialist & & \\
Excessive/redundant antimicrobial spectrum & $0.39(0.18-0.84)$ & 0.02 \\
Age>60 & $2.73(1.34-5.55)$ & 0.006 \\
Prescription on weekends/hollidays & $22.54(7.78-65.30)$ & $<0.001$ \\
Combination therapy & $8.22(3.05-22.5)$ & $<0.001$ \\
Urinary tract infection & $0.65(0.02-0.16)$ & $<0.001$ \\
Quinolone & $0.16(0.04-0.63)$ & 0.008 \\
Aminoglycoside & & \\
Insufficient antimicrobial spectrum & $8.34(2.55-27.31)$ & $<0.001$ \\
Patient from Intensive Care Unit & $0.14(0.03-0.3)$ & 0.01 \\
Previous consult with ID specialist & $91.15(17.88-464.63)$ & $<0.001$ \\
Peritoneal infection & $0.04(0.00-0.54)$ & 0.02 \\
Urinary tract infection & & \\
\hline
\end{tabular}


Factors independently associated with inappropriateness were: prescription on weekends or holidays, patients admitted to Intensive Care Units (ICUs), peritoneal or urinary tract infections, combination therapy with two or more antimicrobials, and therapy with a penicillin or first-generation cephalosporin. A previous consultation with an ID specialist was protective against inappropriate antimicrobial prescription.

Unnecessary antimicrobial prescriptions (category "U”) were more likely to be directed to presumed peritoneal or urinary tract infection, and to involve a firstgeneration cephalosporin. On the other hand, they were less likely to happen after a consultation with an ID specialist.

Prescriptions from weekends/holidays, urinary tract infection and combination therapy were independent predictors for "E/R" category. This error was less likely to occur with patients aged more than 60 , or in prescriptions including quinolones or aminoglycosides.

Admission in ICU and peritoneal infections were independent predictors of prescriptions in the "I" category. Protective factors were: urinary tract infection and a previous consultation with an ID specialist.

\section{DISCUSSION}

In the last two decades, antimicrobial control policies in hospitals changed from predominantly cost-containing measures to quality promotion strategies. ${ }^{29}$ The attempt to optimize antimicrobial use and minimize resistance led to the development of the ASP in Hospital Estadual Bauru ever since it was opened. 
This posed us a paradoxical problem: it was difficult measure the impact of the ASP through the classical "before-and-after" analysis. ${ }^{13,30}$ The present study tried to overcome this problem by performing an extensive analysis of factors related to inappropriate prescriptions.

The proportion of inappropriateness in antimicrobial prescriptions in our hospital was similar to that reported in other studies. ${ }^{31,32}$ However, our study differs from these and other ${ }^{34-36}$ studies in several ways. First, we excluded all antimicrobials prescribed for prophylactic purposes from our analysis. We believe that particularities of prophylactic use of antimicrobials need to be addressed in specific studies. ${ }^{37}$

Although we performed an analysis of factors predictive of general inappropriateness in prescriptions in similar way as in previous reports ${ }^{31-35}$, we decided to refine our analysis, and address three specific errors (categories "U", "E/R" and "I") We found out that generalization can be misleading. Let us consider the case of urinary tract infections. Patients with this diagnosis were more likely to be treated with overly broad-spectrum antimicrobials, or even unnecessarily. Indeed, many of these patients were found to have asymptomatic bacteriuria, and no need for treatment. In an opposite way - but coherently - a diagnosis of urinary tract infection was protective against therapy with insufficient-spectrum antimicrobials. This is a brief example of details that are lost in analysis of "general inappropriateness".

Peritonitis was also associated with a dual pattern of inappropriateness. It was frequently overdiagnosed, but - when present - approached with insufficient 
antimicrobials. We occasionally detected use of a quinolone or aminoglycoside without association with an anti-anaerobe drug - an error too serious to be conceivable in a teaching hospital.

Other findings are noteworthy. First-generation cephalosporins were often indicated for presumed (but non-existing) skin, surgical site and urinary tract infections. Errors concerning the prescription of penicillins were distributed among several categories. Sometimes penicillin-containing prescriptions fell into the "I" category (e.g., penicillin or oxacillin as sole agents for community-acquired pneumonia), but other times it was part of an "E/R" combination (e.g., oxacillin plus cefepime for community-acquired pneumonia, or oxacillin plus aminoglycoside and metronidazole for peritonitis). Aminoglycosides and quinolones, when they were not part of a combination, were more likely to be appropriately prescribed. Finally, there was a worrisome finding: patients from ICUs were more likely to be treated in antimicrobials with insufficient antiinfective spectrum.

The most striking finding from our study was the importance of ID specialist consultations in preventing inappropriate prescriptions: either in general, or for "U" and "I" categories. However significant they may be, these findings are still probably underestimated. In fact, many ID consultations in the study year were made by telephone calls, and could not be traced in our analysis. Indirect evidence points out to an even greater benefit of ID consultations. The "E/R" category was more likely to involve prescriptions made on weekends or holidays, when ID specialists were not available. The foremost 
importance of ID consultation has been extensively demonstrated in previous studies from developed ${ }^{38}$ and developing countries. ${ }^{32,34,35}$ This is a sound argument for the universal importance of ASP as a tool to promote quality of health care.

Some aspects from our study deserve explanation. First, we did not analyze predictors for errors classified in categories "S/L" and "L/H". Since our study was based on "requests" which were often altered by the ASP team, data on dosing and duration reflected - at best - the prescriber's intention. We did not find those data accurate enough to warrant a specific analysis.

One could expect the categorization of antimicrobial use by the authors to be affected by subjectivity. For this reason, we performed analysis independently, and used kappa coefficient to measure inter-rater agreement. The kappa values measure how much the agreement exceeds what would be expected by chance alone. The kappa coefficient from our study $(0.89)$ is rated as "almost complete agreement" in the classic Landis \& Koch classification. ${ }^{39}$

Several changes in ASP policies were instituted after our study: (1) ID consultation on weekends and holidays; (2) ASP daily rounds in ICUs; (3) online request form for antimicrobials; (4) routine reporting all microbiological results to the ASP team. While new studies are needed to quantify the impact of these changes, preliminary analysis suggests an at least three-fold increase in prescriptions based on culture results.

In conclusion, the analysis of factors predictive of inappropriateness in antimicrobial prescription allowed us to identify issues requiring intervention. 
Also, it provided a positive feedback of the ASP efficacy, demonstrating the importance of previous consultation with an ID specialist to assure the quality of antimicrobial prescriptions.

\section{REFERENCES}

1. van der Meer JW, Gyssens IC. Quality of antimicrobial drug prescription in hospital. Clin Microbiol Infect 2001; 7 (suppl 6):12-5.

2. Iannini P, Mandell L, Felmingham J, Patou G, Tillotson GS. Adverse cutaneous reactions and drugs: a focus on antimicrobials. J Chemother 2006;18:127-39.

3. Owens RC Jr. QT prolongation with antimicrobial agents: understanding the significance. Drugs 2004;64:1091-124.

4. Hookman P, Barkin JS. Clostridium difficile associated infection, diarrhea and colitis. World J Gastroenterol. 2009;15:1554-80.

5. Goldmann DA, Weinstein RA, Wenzel RP et al. Strategies to Prevent and Control the Emergence and Spread of Antimicrobial-Resistant Microorganisms in Hospitals. A challenge to hospital leadership. JAMA. $1996 ; 275: 234-40$

6. Safdar N, Maki DG. The commonality of risk factors for nosocomial colonization and infection with antimicrobial-resistant Staphylococcus 
aureus, enterococcus, gram-negative bacilli, Clostridium difficile, and Candida. Ann Intern Med 2002;136:834-44.

7. Tacconelli E. Antimicrobial use: risk driver of multidrug resistant microorganisms in healthcare settings. Curr Opin Infect Dis. 2009;22:3528.

8. Kollef $\mathrm{MH}$. Inadequate antimicrobial treatment: an important determinant of outcome for hospitalized patients. Clin Infect Dis 2000; 31 (suppl 4):S131-8.

9. Ruttimann S, Keck B, Hartmeier C et al. Long-term antibiotic cost savings from a comprehensive intervention program in a medical department of a university-affiliated teaching hospital. Clin Infect Dis 2004; 38: 348-56.

10. Sipahi OR. Economics of antibiotic resistance. Expert Rev Anti Infect Ther 2008;6:523-39.

11. Fishman N. Antimicrobial stewardship. Am J Med. 2006 ;119(suppl 1):S53-61.

12. Dellit TH, Owens RC, McGowan Jr JE, et al. Infectious Diseases Society of America and the Society for Healthcare Epidemiology of America gpuidelines for developing an institutional program to enhance antimicrobial stewardship. Clin Infect Dis 2007; 44:159-77.

13. Ng CK, Wu TC, Chan WM, et al. Clinical and economic impact of an antibiotics stewardship programme in a regional hospital in Hong Kong. Qual Saf Health Care 2008;17:387-92. 
14. Martin C, Ofotokun I, Rapp R, et al. Results of an antimicrobial control program at a university hospital. Am J Health Syst Pharm 2005;62:732-8.

15. Davey $P$, Brown E, Fenelon L, et al. Interventions to improve antibiotic prescribing practices for hospital inpatients. Cochrane Database Syst Rev 2005;4:CD003543.

16. Tunkel AR, Hartman BJ, Kaplan SL, et al. Practice guidelines for the management of bacterial meningitis. Clin Infect Dis 2004;39:1267-84.

17. Mandell LA, Bartlett JG, Dowell SF, et al. Update of practice guidelines for the management of community-acquired pneumonia in immunocompetent adults. Clin Infect Dis 2003; 2003; 37:1405-33.

18. American Thoracic Society, Infectious Diseases Society of America. Guidelines for the management of adults with hospital-acquired, ventilatorassociated, and healthcare-associated pneumonia. Am J Respir Crit Care Med 2005;171:388-416.

19. Guerrant RL, Van Gilder T, Steiner TS, et al. Practice guidelines for the management of infectious diarrhea. Clin Infect Dis. 2001;32:331-51.

20. Solomkin JS, Mazuski JE, Baron EJ et al. Guidelines for the selection of anti-infective agents for complicated intra-abdominal infections. Clin Infect Dis 2003;37:997-1005.

21. Warren JW, Abrutyn E, Hebel JR, et al. Guidelines for antimicrobial treatment of uncomplicated acute bacterial cystitis and acute pyelonephritis in women. Infectious Diseases Society of America (IDSA). Clin Infect Dis1999;29:745-58. 
22. Nicolle LE, Bradley S, Colgan R, et al. Infectious Diseases Society of America guidelines for the diagnosis and treatment of asymptomatic bacteriuria in adults. Clin Infect Dis 2005;40:643-54.

23. Stevens DL, Bisno AL, Chambers HF, et al. Practice guidelines for the diagnosis and management of skin and soft-tissue infections. Clin Infect Dis $2005 ; 41: 1373-406$.

24. Lipsky BA, Berendt AR, Deery HG, et al. Diagnosis and Treatment of diabetic foot infections. Clin Infect Dis 2004; 39:885-910.

25. Kunin CM, Tupasi T, Craig WA. Use of antibiotics. A brief exposition of the problem and some tentative solutions. Ann Intern Med1973;79:555-60.

26. Jones SR, Pannell J, Barks J, et al. The effect of an educational program upon hospital antibiotic use. Am J Med Sci 1977;273:79-85.

27. Roberts C. Modelling patterns of agreement for nominal scales. Stat Med 2008;27:810-30.

28. Greenland S. Modeling and variable selection in epidemiologic analysis. Am J Public Health 1989;79:340-9.

29. MacDougall C, Polk RE. Antimicrobial stewardship programs in health care systems. Clin Microbiol Rev 2005;18:638-56.

30. Apisarnthanarak A, Danchaivijitr S, Khawcharoenporn T, et al. Effectiveness of education and antibiotic-control program in a tertiary care hospital in Thailand. Clin Infect Dis 2006; 42: 768-75. 
31. Willemsen I,, Groenhuijzen A, Bogaers D, et al. Appropriateness of antimicrobial therapy measured by repeated prevalence surveys. Antimicrob Agents Chemother 2007;51:864-7.

32. Erbay A, Colpan A, Bodur $\mathrm{H}$, et al. Evaluation of antibiotic use in a hospital with an antibiotic restriction policy. Int $\mathrm{J}$ Antimicrob Agents 2003;21:308312.

33. Tünger O, Dinç G, Özbakkaloglu B, et al. Evaluation of rational antibiotic use. Int J Antimicrob Agents 2000;15:131-5.

34. Erbay A, Bodur $\mathrm{H}$, Akinci $\mathrm{E}$, et al. Evaluation of antibiotic use in intensive care units of a tertiary care hospital in Turkey. J Hosp Infect. 2005;59:5361.

35. Apisarnthanarak A, Danchaivijitr S, Bailey TC, et al. Inappropriate antibiotic use in a tertiary care center in Thailand: an incidence study and review of experience in Thailand. Infect Control Hosp Epidemiol 2006;27:416-20.

36. Hecker MT, Aron DC, Patel NP, et al. Unnecessary use of antimicrobials in hospitalized patients. Arch Intern Med 2003;163:972-8.

37. Tan JA, Naik VN, Lingard L. Exploring obstacles to proper timing of prophylactic antibiotics for surgical site infections. Qual Saf Health Care. 2006;15:32-8.

38. Raineri E, Pan A, Mondello P, et al. Role of the infectious diseases specialist consultant on the appropriateness of antimicrobial therapy prescription in an intensive care unit. Am J Infect Control 2008;36:283-90. 
39. Landis JR, Koch GG. The measurement of observer agreement for categorical data. Biometrics 1977;33:159-74. 